\title{
The Nocardia cyriacigeorgica GUH-2 genome shows ongoing adaptation of an environmental Actinobacteria to a pathogen's lifestyle
}

Anthony Zoropogui ${ }^{1}$, Petar Pujic ${ }^{2}$, Philippe Normand ${ }^{2}$, Valérie Barbe ${ }^{4}$, Patrick Belli ${ }^{3}$, Arnault Graindorge ${ }^{1}$ David Roche ${ }^{4}$, David Vallenet ${ }^{4}$, Sophie Mangenot ${ }^{4}$, Patrick Boiron ${ }^{1,3}$, Véronica Rodriguez-Nava ${ }^{1,3}$, Sebastien Ribun ${ }^{1,3}$, Yves Richard ${ }^{1,4}$, Benoit Cournoyer ${ }^{1,4}$ and Didier Blaha $a^{1,3^{*}}$

\begin{abstract}
Background: Nocardia cyriacigeorgica is recognized as one of the most prevalent etiological agents of human nocardiosis. Human exposure to these Actinobacteria stems from direct contact with contaminated environmental matrices. The full genome sequence of $\mathrm{N}$. cyriacigeorgica strain $\mathrm{GUH}-2$ was studied to infer major trends in its evolution, including the acquisition of novel genetic elements that could explain its ability to thrive in multiple habitats.

Results: N. cyriacigeorgica strain GUH-2 genome size is $6.19 \mathrm{Mb}$-long, $82.7 \%$ of its CDS have homologs in at least another actinobacterial genome, and $74.5 \%$ of these are found in N. farcinica. Among N. cyriacigeorgica specific CDS, some are likely implicated in niche specialization such as those involved in denitrification and RuBisCO production, and are found in regions of genomic plasticity (RGP). Overall, 22 RGP were identified in this genome, representing $11.4 \%$ of its content. Some of these RGP encode a recombinase and IS elements which are indicative of genomic instability. CDS playing part in virulence were identified in this genome such as those involved in mammalian cell entry or encoding a superoxide dismutase. CDS encoding non ribosomal peptide synthetases (NRPS) and polyketide synthases (PKS) were identified, with some being likely involved in the synthesis of siderophores and toxins. COG analyses showed this genome to have an organization similar to environmental Actinobacteria.
\end{abstract}

Conclusion: N. cyriacigeorgica GUH-2 genome shows features suggesting a diversification from an ancestral saprophytic state. GUH-2 ability at acquiring foreign DNA was found significant and to have led to functional changes likely beneficial for its environmental cycle and opportunistic colonization of a human host.

Keywords: Nocardia cyriacigeorgica, Regions of genomic plasticity, Insertion sequences, COG, Evolution, Opportunistic pathogen

\section{Background}

Nocardia is part of the well-known CMN actinobacterial group that also includes Corynebacterium and Mycobacterium in the Corynebacteriales order [1]. These Actinobacteria are characterized by long-chain mycolic acids in their cell wall [2], making them acid-resistant

\footnotetext{
* Correspondence: didier.blaha@univ-lyon1.fr

'Research group on "Bacterial Opportunistic Pathogens and Environment", Université de Lyon, Lyon, France

${ }^{3}$ Research group on "Environmental Microbiology Lyon - Biological Resource Center", UMR5557 Ecologie Microbienne, Université de Lyon, Université Lyon 1, CNRS, and VetAgro Sup Veterinary Campus, Lyon, France

Full list of author information is available at the end of the article
}

according to the Ziehl-Neelsen staining procedure, and favoring resistance to hydrophilic chemicals and dehydration. All CMN genera include pathogenic strains causing human diseases that affect millions of individuals such as leprosy, tuberculosis, and diphtheria. Besides, the CMN group also includes saprophytes that thrive in soils, waters, and polluted environments.

The Nocardia genus comprises about 80 species [3]. $N$. cyriacigeorgica was defined as a species in 2001 following the isolation and characterization of strain IMMIB D$1627 \mathrm{~T}$ from a bronchial discharge in a chronic bronchitis patient in Gelsenkirchen, Germany [4]. N. cyriacigeorgica

\section{() Biomed Central}


can be differentiated from other species by $16 \mathrm{~S}$ rDNA sequence analysis, their ability at growing on acetamide but inability at using proline as carbon and nitrogen sources. Definition of this species was confirmed by Conville et al. (2007) during their investigation of Nocardia strains with a type VI drug resistance pattern (characterized by a resistance to penicillins and a susceptibility to the broadspectrum cephalosporins) [5]. N. cyriacigeorgica differs from $N$. farcinica strains by their ability at synthesizing a nitrate reductase and hydrolyzing xantine but their inability at synthesizing a urease, at hydrolyzing esculin and growing on L-rhamnose [6]. N. cyriacigeorgica strains were described as etiological agents of human pneumonia, brain abscesses, and kidney, heart and eye infections [4,7-12]. It is the most prevalent species involved in human nocardiosis in North America [11,13] and its prevalence in France was estimated at 12\% among human nocardial infections declared between 2000 and 2007 [14]. Nocardiosis can be fatal for immunosuppressed individuals $[13,14]$. There is no report of Nocardia crosscontaminations in human populations, suggesting that environmental exposure is the main cause of infection. However, $N$. cyriacigeorgica has rarely been reported among environmental samples. Nevertheless, $N$. cyriacigeorgica strains have been recovered from oil contaminated soils $[15,16]$, and were shown to oxidize a variety of aliphatic compounds [17].

In this work, the $N$. cyriacigeorgica GUH-2 genome sequence is presented, and compared with those of other Actinobacteria. The GUH-2 strain was isolated from a primary human kidney infection with systemic progression, which had a fatal outcome at Georgetown University Hospital, Washington, D.C [10]. The ability of this strain to induce Parkinson-like symptoms in inoculated mouse and monkey models $[18,19]$ made it the model strain to study Nocardia biology and pathogenesis. These latter observations led to investigations on its possible role in some human Parkinson cases [20,21]. Animals infected by $N$. cyriacigeorgica were found to develop abnormal behaviors like rhythmic vertical "yes-yes" head-shaking movements, stooped posture, hesitation to move forward, retropulsion, and restlessness [22]. These parkinsonian-like symptoms appeared to be related to (i) a decrease in dopamine receptors and (ii) a programmed cell death of dopaminergic neurons within the substantia nigra in mice [23]. Intraperitoneal injection of antiparkinsonian drugs such as LDOPA temporarily alleviated these symptoms $[18,24]$. $N$. cyriacigeorgica GUH-2 was also reported, in several independent experiments, to produce a substance(s) that can induce apoptosis and dopamine depletion [25,26]. Inferences on the likely nature of this substance(s) (probably a proteasome inhibitor) were made from the CDS sequence presented in this paper. A transposon mutagenesis screening of Mycobacterium tuberculosis showed the structural proteasomal genes of this closely related bacterium to be involved in their response toward oxidative and nitrosative stresses [27]. The nocardial proteasome could thus play a role in virulence.

The GUH-2 genomic sequence was also used to identify key evolutionary events in the emergence of $N$. cyriacigeorgica. Other members of the CMN group were shown to have evolved through important DNA reshuffling. Insertion sequences (IS) have largely contributed to genome rearrangements in the Corynebacterium and Mycobacterium genera, favoring deletion of genes, inversions, and functional specialization [28]. Important levels of CDS duplications and domains reshuffling were reported in Mycobacteria (50\% of M. tuberculosis H37Rv) [29]. Statistical tests were performed to identify biases in N. cyriacigeorgica GUH-2 CDS and COG contents. A phylogenomic approach was developed to track the origin of some CDS or CDS clusters. These comparisons highlighted regions of genomic plasticity (RGP) among the $N$. cyriacigeorgica GUH-2 genome. Selection of these RGP was probably a driving force in the emergence of $N$. cyriacigeorgica GUH-2. These results revealed highly dynamic genomic evolutionary patterns in $N$. cyriacigeorgica caused by a significant ability at acquiring foreign DNA.

\section{Results}

\section{Virulence status of $\mathrm{N}$. cyriacigeorgica GUH-2}

Virulence of $N$. cyriacigeorgica GUH-2 strain was confirmed by intravenous injection of approximately $10^{7} \mathrm{CFU}$ in the tail of BALB/c mice. The death rate rose to $60 \% 7$ days after infection. Autopsy indicated death to be due to septicemia with formation of nodules in several organs: kidneys, liver, brain, spleen, lungs and heart (Figure 1). Microscopic analysis of nodules showed high numbers of poly- and mono-nucleated inflammatory

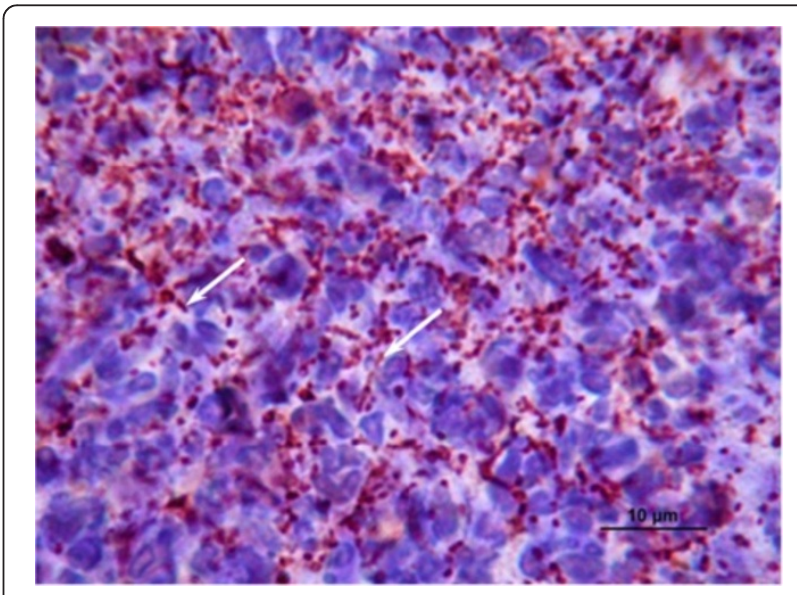

Figure 1 Histological observations of mice tissue infected by N. cyriacigeorgica GUH-2. Photograph illustrating the immunohistochemistry analysis of kidney cells from a case of fatal septicemia; white arrows indicate filamentous bacteria. 
cells, and $N$. cyriacigeorgica GUH-2 cells. Some mice were injected a lower number $\left(3.5 \times 10^{5} \mathrm{CFU}\right)$ of $N$. cyriacigeorgica GUH-2 cells and developed abnormal behavior: rigidity, stooped posture, hemiparesis and vertical yes-yes head shaking probably due to a brain infection.

\section{General features of the Nocardia cyriacigeorgica genome}

The 6.19-Mb genome of $N$. cyriacigeorgica was sequenced and annotated during this work. It was found to harbor a single circular chromosome, and to have a $\sim 68.4 \% \mathrm{G}+\mathrm{C}$ content. Three $r r n$ operons containing the genes for the 16S, $23 \mathrm{~S}$ and 5S rRNAs, 5,477 predicted protein-coding sequences (CDS), 49 tRNA genes, and 14 pseudogenes i.e. truncated genes, were detected. A function could be predicted for most CDS (62.23\%). Other CDS were detected among other bacterial groups $(28.10 \%)$ or showed no homology with known sequences (9.67\%) (Table 1). $N$. cyriacigeorgica GUH-2 strain did not harbor a plasmid. The genome coding density was estimated at $86.73 \%$, which is markedly lower than the $91 \%$ value observed in related genomes (Table 1).

GUH-2 chromosome harbors eleven insertion sequences (see IS section), and 15 CDS encoding putative phage proteins. Putative virulence genes are scattered along the chromosome without distinguishable pathogenicity island (see Additional file 1 for a complete listing). Several syntons of 5 CDS or more were found conserved between $N$. cyriacigeorgica, N. farcinica, Rhodococcus jostii and Mycobacterium tuberculosis (Figure 2). Non-conserved regions frequently showed distinct $\mathrm{G}+\mathrm{C} \%$ biases. Regions encoding the largest putative proteins from the genome were found to have features of non ribosomal peptide synthetases (NRPS) and showed a high $\mathrm{G}+\mathrm{C}$ content. The only exception is NOCYR_4710 CDS which has a G + C content slightly lower than the averaged one, and encodes

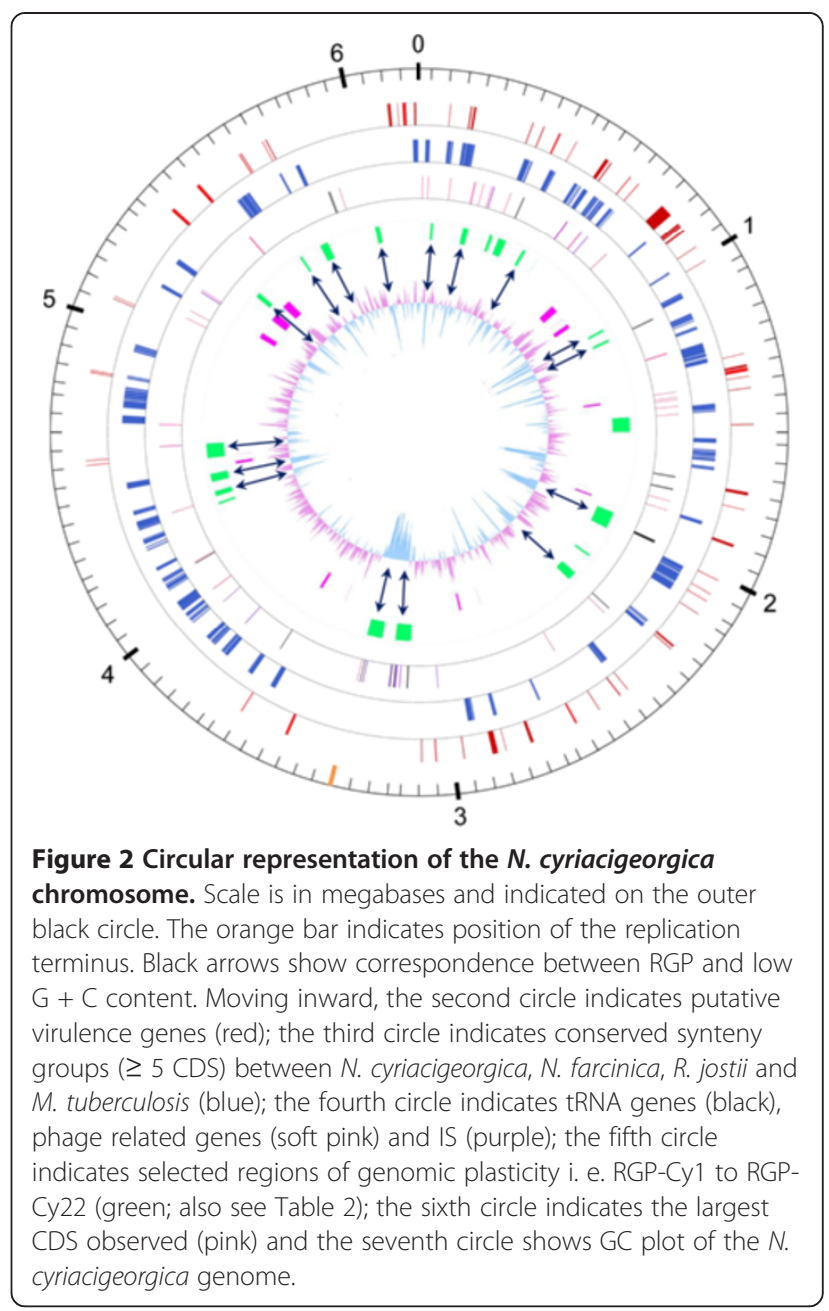

Table 1 Comparison of genomic features between $N$. cyriacigeorgica GUH-2 and eight Actinobacteria

\begin{tabular}{|c|c|c|c|c|c|c|c|c|c|}
\hline \multirow{3}{*}{ Features } & \multirow{3}{*}{$\begin{array}{c}N . \\
\frac{\text { cyriacigeorgica }}{\text { GUH-2 }}\end{array}$} & \multirow{3}{*}{$\frac{\begin{array}{c}N . \\
\text { farcinica }\end{array}}{10152}$} & \multirow{3}{*}{$\begin{array}{l}\text { R. equi } \\
\text { ATCC } \\
33707\end{array}$} & \multirow{3}{*}{$\frac{R . \text { jostii }}{\text { RHA1 }}$} & \multirow{3}{*}{$\begin{array}{c}\text { M. } \\
\frac{\text { tuberculosis }}{\text { H37Rv }}\end{array}$} & \multirow{3}{*}{$\begin{array}{c}\text { M. } \\
\text { smegmatis } \\
\text { MC2 } 155\end{array}$} & \multirow{3}{*}{$\begin{array}{c}\text { C. } \\
\text { diphtheriae } \\
\text { NCTC } \\
13129\end{array}$} & \multirow{3}{*}{$\begin{array}{c}C . \\
\frac{\text { glutamicum }}{\text { ATCC }} \\
13032\end{array}$} & \multirow{3}{*}{$\begin{array}{c}\begin{array}{c}A . \\
\text { mediterranei }\end{array} \\
\mathrm{U} 32\end{array}$} \\
\hline & & & & & & & & & \\
\hline & & & & & & & & & \\
\hline Size (nt) & $6,194,645$ & $6,021,225$ & $5,235,298$ & $9,702,737$ & $4,411,532$ & $6,988,209$ & $2,488,635$ & $3,309,401$ & $10,236,715$ \\
\hline G + C (\%) & 68.37 & 70.83 & 68.81 & 67.0 & 65.61 & 67.40 & 53.48 & 53.81 & 71.29 \\
\hline Average CDS length (nt) & 983.29 & 922.77 & 952.39 & 872.17 & 923.13 & 905.65 & 927.24 & 923.69 & 936.1 \\
\hline $\begin{array}{l}\text { Average intergenic region } \\
\text { (nt) }\end{array}$ & 171.09 & 117.23 & 109.07 & 117.54 & 114.69 & 84.84 & 119.08 & 156.45 & 126.77 \\
\hline $\begin{array}{l}\text { Protein coding density } \\
(\%)\end{array}$ & 86.73 & 90.93 & 91.16 & 90.29 & 91.33 & 91.16 & 87.11 & 86.49 & 90.69 \\
\hline $\begin{array}{l}\text { Protein-coding sequences } \\
\text { (CDS) }\end{array}$ & 5.491 & 5.984 & 5124 & 9.145 & 4.454 & 7.449 & 2.491 & 3.128 & 9.988 \\
\hline Pseudogenes & 14 & $U n^{*}$ & 13 & 40 & 8 & 290 & 131 & 24 & \\
\hline rRNA (operon) & 3 & 3 & 1 & 4 & 3 & 6 & 5 & 6 & 4 \\
\hline tRNA & 49 & 53 & 52 & 52 & 45 & 47 & 54 & 60 & 52 \\
\hline
\end{tabular}


a putative Dipeptidyl carboxypeptidase Dcp located near a transposase-related CDS (Figure 2).

To better understand the events that had led to the present-day $N$. cyriacigeorgica GUH-2 genomic structure, its core genome was delimited by identifying CDS conserved in a panel of closely related Actinobacteria (Figure 3). 15\% (805 of 5477) of the CDS was found in a putative common ancestor to all genomes except Frankia sp. CcI3 (Figure 4). Of these CDS, 80\% could be assigned a function and, as expected, a large proportion was inferred to play part in basic bacterial functions such as synthesis of proteins, nucleosides and nucleotides, amino acids, co-factor prosthetic groups and carriers, and of the cell envelope (see Additional file 2 for the full listing). Interestingly, the only CDS exclusively shared between $N$. cyriacigeorgica and M. tuberculosis were those of IS987, an insertion sequence, suggesting a likely transfer of this IS between these species. The $N$. cyriacigeorgica and $N$. farcinica genomes were found to share $74.5 \%$ of their CDS, delimiting a Nocardia pangenome of about 4.5 Mb. 1398 CDS (25.5\%) of N. cyriacigeorgica were not found in the $N$. farcinica genome. Most of these CDS are of unknown function (78\%) but some are likely involved in phosphonate transport and metabolism (phytase), synthesis of fatty acids, glutamate metabolism, nitrite/nitrate transport, and RuBisCO production. Conversely, 2253 CDS of $N$. farcinica were not found in the $N$. cyriacigeorgica genome (data not shown). Again, a high proportion of these CDS (69\%) were "unk" CDS (unknown function) and a few could be related to particular activities like synthesis of thiocyanate (toxic compound) (4 CDS), catabolism of urea (6 CDS), of auxins (6 CDS) and lignin, heavy metal resistance and virulence ( $9 \mathrm{CDS})$. These differences were in line with biochemical tests such as measurement of nitrate reductase and urease activities, performed to differentiate these two species. 11.6\% CDS (633 out of 5477) of $N$.

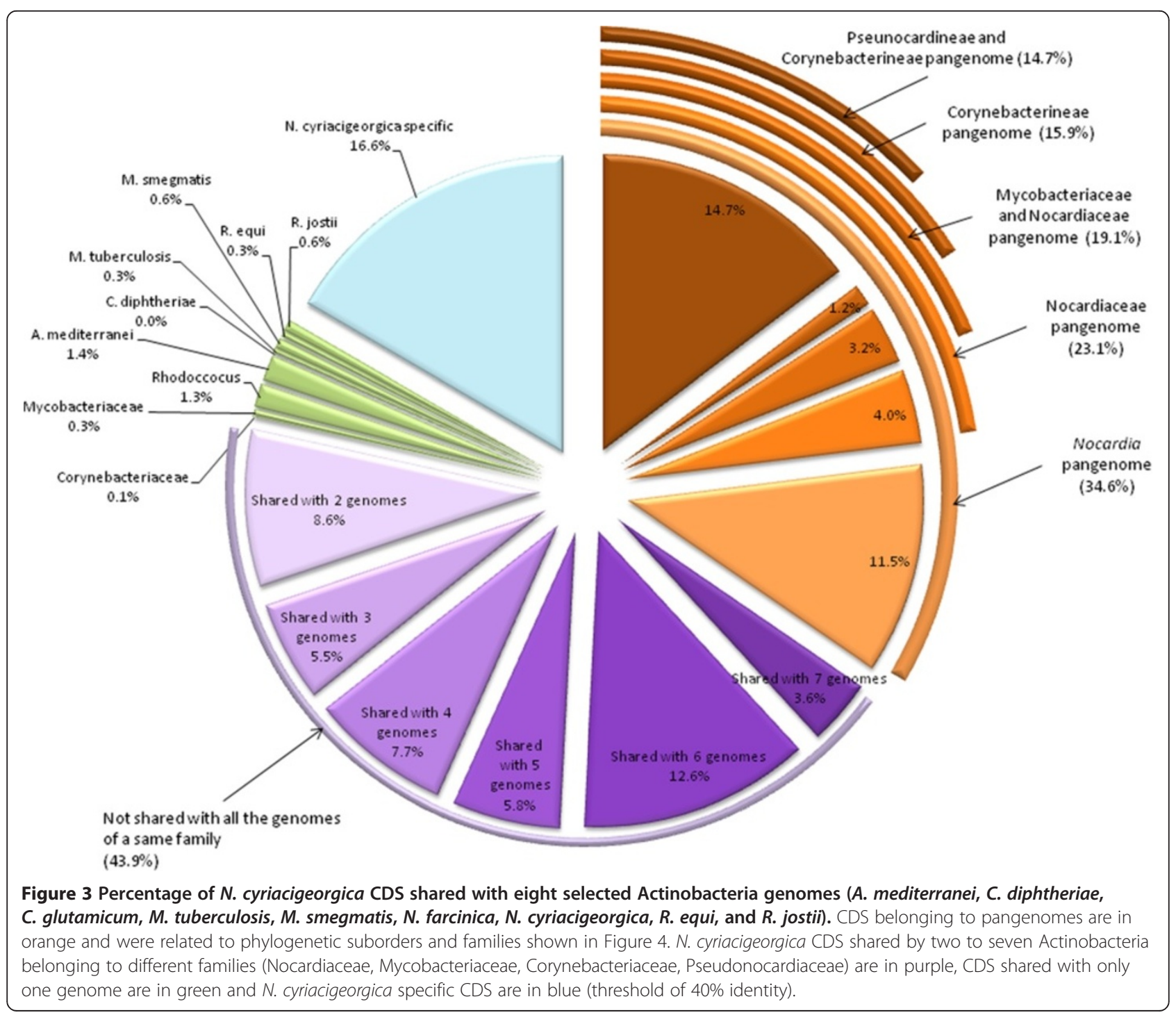




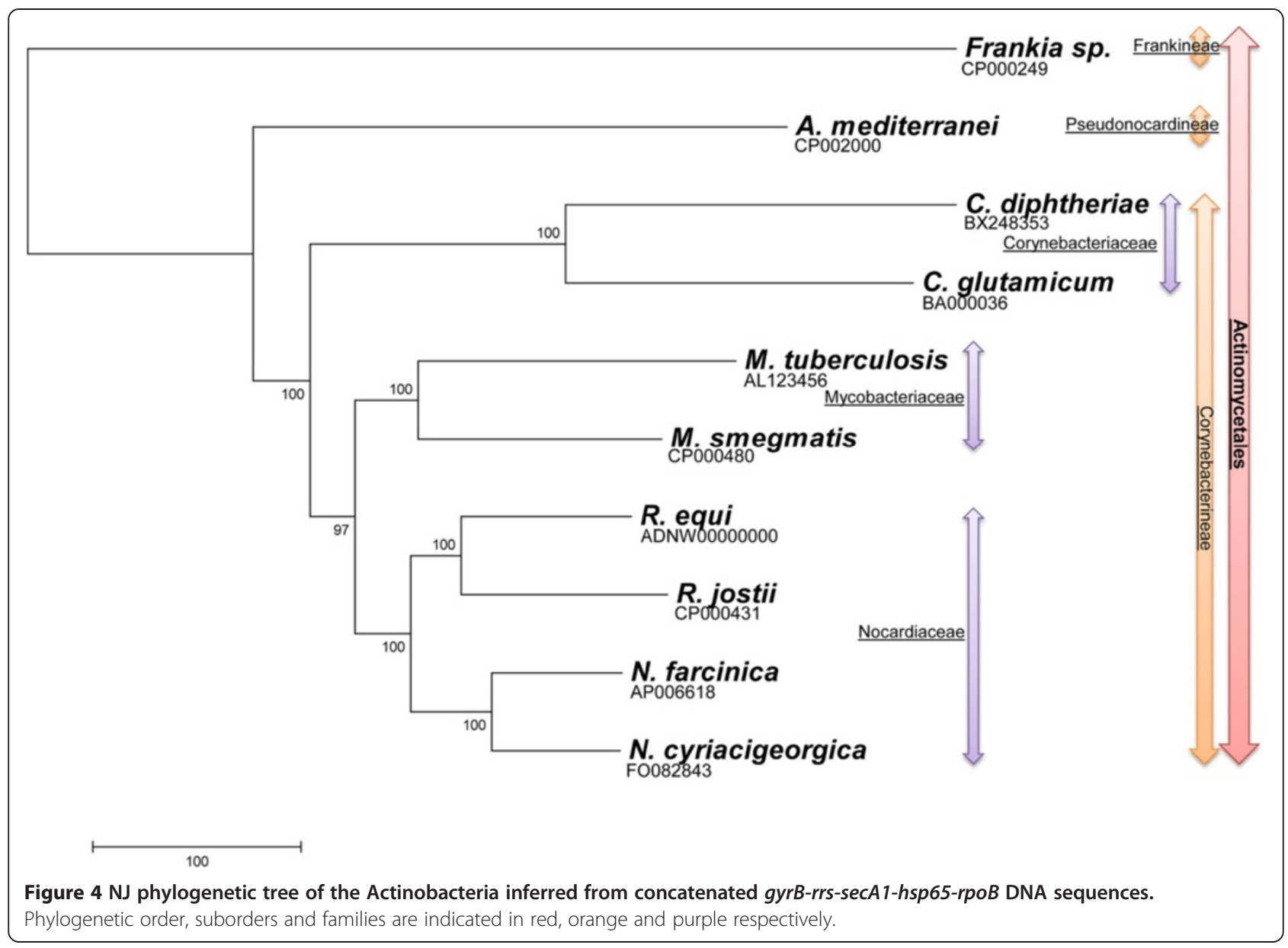

farcinica and $N$. cyriacigeorgica genomes were not recorded in other actinobacterial sequenced genomes (Figure 3). These two Nocardia strains have an equivalent genome size which is longer than the ones of primary actinobacterial pathogens (Table 1). 16.6\% (777 kb) of $N$. cyriacigeorgica CDS content was not recorded in any other organisms referenced in the databases. These CDS were sometimes related to mobile and extrachromosomal elements but most of them were of "unknown function". A search for amplified CDS among $N$. cyriacigeorgica genome revealed 161 occurrences, of which 132 are duplicated, 21 are triplicated and 8 are quadruplicated. More than 29\% of these CDS could not be given a particular function, and $79 \%$ were also found in $N$. farcinica. IS (1 CDS), mce (4 CDS), transcriptional regulators $(12 \mathrm{CDS})$ and nitrate reductase (6 CDS) were part of these amplified regions. More than $23 \%$ of these CDS were found among regions of genomic plasticity (RGP) (see Additional file 3 for more details).

Out of the 5477 CDS present in $N$. cyriacigeorgica genome, 4016 CDS (i. e. 73\%) could be assigned to a COG [see Additional file 4]. The proportion of these COGs among Actinobacteria was similar, with a slightly higher occurrence of CDS among the "transcription" (K) and "signal transduction" (T) COGs of the Nocardia genomes. Correspondence analysis of the number of CDS per COG among a set of actinobacterial species was performed, to identify a possible bias related to the pathogenic nature of the species. This analysis showed that the number of CDS per COG could differentiate primary pathogens from non-pathogens. This "pathogen pattern" was more significant than the COG organization bias inferred from species belonging to a same genus. On the other hand, all non-pathogens had closer COG patterns even though some were part of different genus or part of a genus showing pathogenic species. COG patterns of the Nocardia genomes were found similar to those of non-pathogens (Figure 5, but also see the Additional file 4). A correspondence analysis on the functional domains inferred from the CDS of the "transcription" COG was performed (Additional file 5 and Additional file 6). This analysis did not segregate the dataset according to the pathogenic nature of the species regardless of their genus. Instead, the numbers of CDS per sub-division were found similar between the Nocardia genomes and similar to those observed among $M$. tuberculosis. 


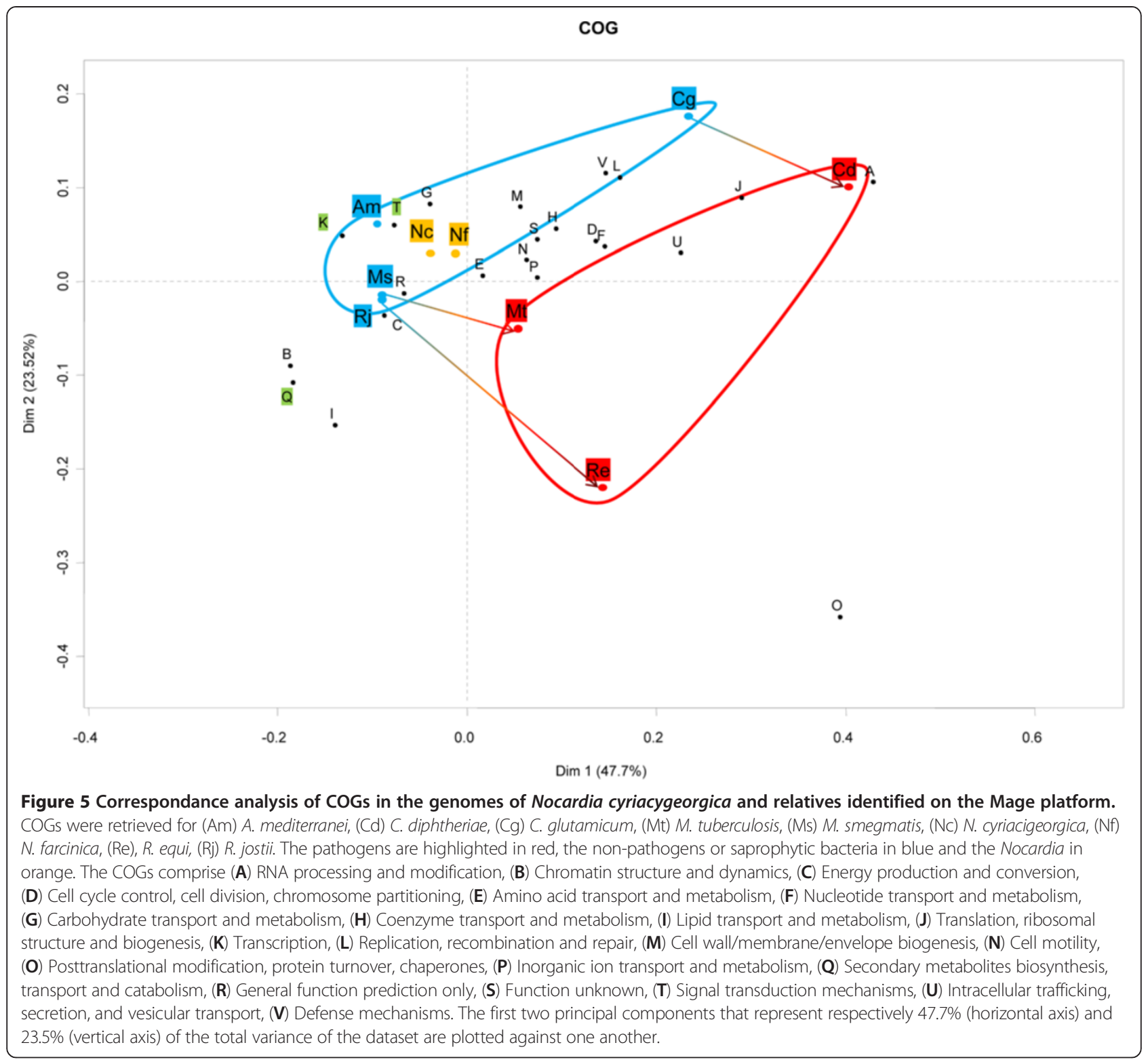

\section{Regions of genomic plasticity}

A lineplot graph between $N$. farcinica and $N$. cyriacigeorgica genomes, representing synteny results of series of 5 CDS or more, was performed in order to visualize the distribution of variable and conserved regions (Figure 6). This analysis showed the number of conserved CDS between these chromosomes to increase towards their respective origin of replication. The lowest concentration of these CDS was observed around the chromosomal terminus of replication. Overall, the organization of these variations was quite similar between the two halves of the circular chromosome creating a mirror-like effect indicative of increasing evolutionary constraints from the terminus towards the origin of replication (on both strand). The chromosomal terminus is partially visible on the circular map of $N$. cyriacigeorgica genome, and shows a large segment with a distinct $\mathrm{G}+\mathrm{C}$ content according to the GC plot (Figure 2). The Artemis Comparison Tool (ACT) was used to refine these analyses and identify DNA segments $>4.5 \mathrm{~kb}$ or containing more than 5 CDS. These DNA regions were not showing all the features of genomic islands such as a tRNA gene at one end, an integrase CDS or a $\mathrm{G}+\mathrm{C}$ bias distinct from the one of the genome. Twenty-two RGP could be detected using this approach (named RGP-Cy\#) (Table 2). Twentyone of these RGP were also detected with the RGPfinder tool of the MaGeplateform. RGP-Cy6 was not detected in this latter analysis because of its length of $4.9 \mathrm{~kb}$. Of these RGP, four could be considered genomic islets $(<10 \mathrm{~kb})$, and the largest RGP was of about $80 \mathrm{~kb}$. These RGP represented a total of $704 \mathrm{~kb}$ i.e. $11.4 \%$ of the genome and encoded 622 


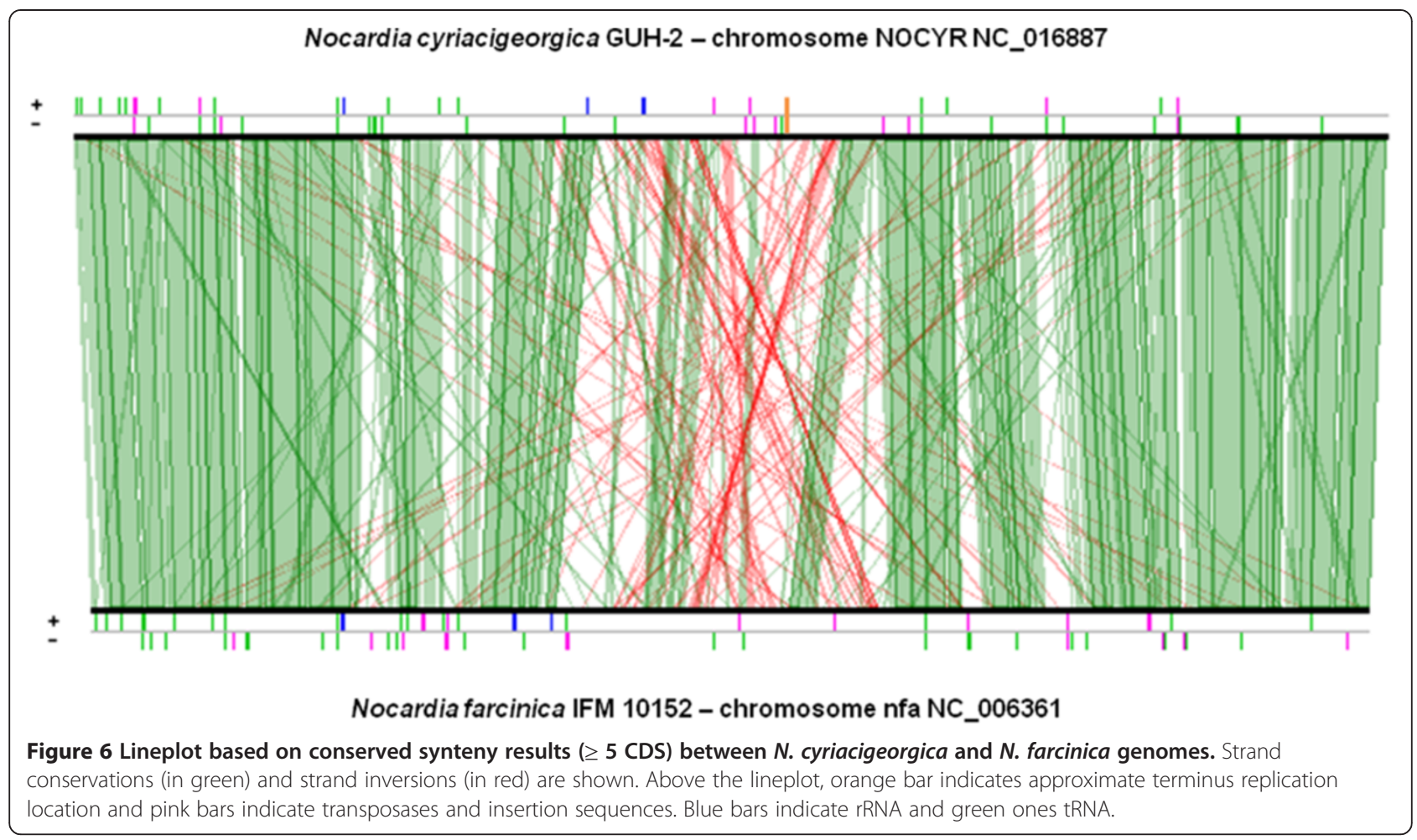

CDS. The average $\mathrm{G}+\mathrm{C} \%$ content of these RGP is of $65.5 \%$, with values ranging from $60.1 \%$ to $68.6 \%$. RGP boundaries were analyzed. tRNA or tmRNA genes were detected at the extremity of ten of these. Direct DNA repeats were observed for RGP-Cy8 from positions 1098658 to 1098672 and 1109676 to 1109690 , at its left and right ends, respectively. Four RGP showed IS sequences. Five RGP contain CDS implicated in DNA modification processes such as integrases, recombinases, endonucleases and excisionases. These CDS were probably involved in the acquisition of these RGP. Ten RGP did not show any of the above features. Most CDS on these selected RGP encode putative proteins and transcriptional regulators of unknown function (74\%). Some CDS, likely representing a benefit for N. cyriacigeorgica GUH-2, could be identified and predicted to encode a catalase (RGP-Cy3), a limonene monooxygenase (RGP-Cy16), and a sulfonate ABC transporter (RGP-Cy21). Furthermore, CDS involved in nitrate metabolism were identified on the RGP-Cy14. A cobalamin-independent methionine synthase (metE) and three CDS involved in citrate metabolism were found on RGP-Cy15 while $p g l Y$ and $p g l Z$ involved in phage defense were found on Cy10 (Table 2).

PCR screenings were designed to investigate the distribution of these RGP among $83 \mathrm{~N}$. cyriacigeorgica strains (Additional file 7). Prevalence of these RGP was quite variable, with some not being detected in other strains, and some being found among up to $69 \%$ of the strains. RGP-Cy4 and RGP-Cy8 were only found in the $N$. cyriacigeorgica GUH-2 genome and showed all the features of mobile elements. A cladogram was built using the RGP distribution patterns based on the positive and negative PCR results (Figure 7). Dataset of inner RGP and RGP-ends DNA targets were analyzed separately or together, and gave similar cladograms. All strains harboring 5 or more RGP were grouped in the "GUH-2 complex". Other strains belonged to a "type strain complex". In fact, RGP patterns of 30 strains were found to be in the "GUH-2 complex", while patterns of 56 strains were allocated to the "type strain complex". Among the "GUH-2 complex", some of the selected RGP were highly prevalent: RGP-Cy1, RGP-Cy6, RGP-Cy11, RGPCy15, RGP-Cy16, RGP-Cy18 and RGP-Cy21 were found in $23,18,25,29,23,20$, and 26 strains respectively. Strain N7 harbored the highest number of RGP found in the $N$. cyriacigeorgica GUH-2 genome (15/22 positive PCR products). Among the type strain complex, RGPСy2, -Сy5, -Сy6, -Сy7, -Сy9, -Сy10, -Сy16, -Сy18, -Сy19, - Cy20, and -Cy21 were not recorded. No RGP seemed to be representative of this latter complex, and RGP-Cy3, RGP-Cy15 and RGP-Cy17 were the most prevalent. RGPCy3 was the most broadly distributed (69 positive strains) among the $N$. cyriacigeorgica species. PCR screenings targeting the left and right ends of RGP were defined to estimate the level of conservation of their respective proximal DNA region. The left (L) and right (R) ends of RGP-Cy9, Cy15, and -Cy16 were broadly detected whereas only one 
Table 2 Regions of genomic plasticity (RGP-Cy\#) identified in N. cyriacigeorgica by ACT comparisons with the $\mathbf{N}$. farcinica genome

\begin{tabular}{|c|c|c|c|c|c|c|c|c|c|}
\hline RGP & Gl position & $\begin{array}{l}\text { Virulence gene } \\
\text { (in or around Gl) }\end{array}$ & $\begin{array}{l}\text { ORF } \\
\text { begin }\end{array}$ & ORF end & $\begin{array}{l}\text { Length } \\
(\mathrm{kb})\end{array}$ & $\begin{array}{l}\text { Nb. of } \\
\text { genes }\end{array}$ & Gene class & Putative role of $\mathrm{Gl}$ & tRNA \\
\hline Cy1 & $61471 . .73200$ & & nocyr_0048 & nocyr_0063 & 11.7 & 14 & Unknown & Unknown & 0 \\
\hline Cy2 & $213775 . .239600$ & & nocyr_0182 & nocyr_0205 & 25.8 & 21 & $\begin{array}{l}\text { Enzymes, transporters, } \\
\text { histidine degradation, CSP }\end{array}$ & Unknown & 3 \\
\hline Суз & $343550 . .357558$ & nocyr_0300 & nocyr_0300 & nocyr_0309 & 14 & 10 & $\begin{array}{l}\text { Enzymes, regulators, } \\
\text { transporters, catalase }\end{array}$ & $\begin{array}{l}\text { Adaptation to } \\
\text { atypical conditions }\end{array}$ & 1 \\
\hline Cy4 & $387100 . .424100$ & & nocyr_0340 & nocyr_0383 & 37 & 43 & $\begin{array}{l}\text { Recombinase, integrase, } \\
\text { topisomerase, ADN pol III, } \\
\text { cadmium inductible } \\
\text { protein }\end{array}$ & $\begin{array}{l}\text { Plasticity, } \\
\text { adaptation }\end{array}$ & 1 \\
\hline Cy5 & $512800 . .526200$ & & nocyr_0472 & nocyr_0488 & 13.4 & 17 & regulators, transporters & Unknown & 0 \\
\hline Cy6 & $594300 . .599228$ & $\begin{array}{l}0547 \rightarrow 0555 \\
0566 \rightarrow 0567\end{array}$ & nocyr_0557 & nocyr_0561 & 4.9 & 5 & Regulators, ISNcy5 & Plasticity & 1 \\
\hline Cy7 & $1045000 . .1055000$ & & nocyr_0920 & nocyr_0925 & 10 & 6 & Unknown & Unknown & 1 \\
\hline Cy8 & $1098658 . .1109690$ & & nocyr_0967 & nocyr_0978 & 11.3 & 11 & $\begin{array}{l}\text { Integrase, } \\
\text { endoribonuclease }\end{array}$ & Plasticity & 0 \\
\hline Cy9 & $1480355 . .1548800$ & & nocyr_1341 & nocyr_1391 & 69.3 & 51 & $\begin{array}{l}\text { Enzymes, regulators, } \\
\text { mycosin }\end{array}$ & Virulence & 2 \\
\hline Cy10 & $1937100 . .2016932$ & nocyr_1792 & nocyr_1739 & nocyr_1800 & 79.8 & 68 & $\begin{array}{l}\text { Enzymes, regulators, C } 31 \\
\text { phage resistance genes }\end{array}$ & Defense & 1 \\
\hline Cy11 & $2164650 . .2173100$ & & nocyr_1932 & nocyr_1940 & 8.4 & 9 & Unknown & Unknown & 0 \\
\hline Cy12 & $2273150 . .2315500$ & & nocyr_2044 & nocyr_2082 & 42.4 & 36 & $\begin{array}{l}\text { Enzymes, transporters, } \\
\text { regulators, integrases }\end{array}$ & Plasticity & 1 \\
\hline Cy13 & 3131000.3204200 & & nocyr_2827 & nocyr_2884 & 73.2 & 58 & $\begin{array}{l}\text { Enzymes, regulators, } \\
\text { ISNcy2-b, ISNcy4, } \\
\text { integrases, PBP }\end{array}$ & $\begin{array}{l}\text { Plasticity, } \\
\text { adaptation }\end{array}$ & 0 \\
\hline Cy14 & 3265400.3337600 & & nocyr_2933 & nocyr_2985 & 72.2 & 61 & $\begin{array}{l}\text { Enzymes, Nitrate reduction/ } \\
\text { expulsion, ISNcy1-d, } \\
\text { regulators, recombinase }\end{array}$ & $\begin{array}{l}\text { Nitrite/Nitrate } \\
\text { metabolism, } \\
\text { plasticity }\end{array}$ & 1 \\
\hline Cy15 & $4299671 . .4308500$ & & nocyr_3906 & nocyr_3911 & 8.8 & 6 & $\begin{array}{l}\text { Cobalamin and citrate } \\
\text { metabolism }\end{array}$ & $\begin{array}{l}\text { Vit B12, energy } \\
\text { metabolism }\end{array}$ & 0 \\
\hline Cy16 & $4339344 . .4363500$ & & nocyr_3940 & nocyr_3962 & 24.2 & 23 & $\begin{array}{l}\text { Enzymes, regulators, } \\
\text { limonene-momooxygenase }\end{array}$ & $\begin{array}{l}\text { Unknown, } \\
\text { Adaptation }\end{array}$ & 0 \\
\hline Cy17 & $4409577 . .4447497$ & & nocyr_4002 & nocyr_4035 & 37.9 & 34 & $\begin{array}{l}\text { Enzymes, regulators, } \\
\text { transporters }\end{array}$ & Unknown & 0 \\
\hline Cy18 & 4523400.4592400 & $\begin{array}{l}\text { nocyr_4112, } \\
\text { nocyr_4128 }\end{array}$ & nocyr_4097 & nocyr_4162 & 69 & 66 & $\begin{array}{l}\text { Enzymes, regulators, NRPS, } \\
\text { transporters, fatty acid } \\
\text { synthesis, spermidin, sigma } \\
\text { factor, DHB }\end{array}$ & $\begin{array}{l}\text { Fatty acid and } \\
\text { peptide synthesis, } \\
\text { adaptation, } \\
\text { virulence, plasticity }\end{array}$ & 1 \\
\hline Cy19 & $5338862 . .5361100$ & & nocyr_4801 & nocyr_4818 & 22.2 & 14 & Enzymes, regulators & $\begin{array}{l}\text { Unknown, vitamine } \\
\text { métabolism }\end{array}$ & 0 \\
\hline Cy20 & 5611900.5622135 & & nocyr_5027 & nocyr_5036 & 10.2 & 10 & Unknown & Unknown & 0 \\
\hline Cy21 & 5720300.5758400 & $\begin{array}{l}\text { nocyr_5135, } \\
\text { nocyr_5136 }\end{array}$ & nocyr_5133 & nocyr_5170 & 38.1 & 38 & $\begin{array}{l}\text { Enzymes, transporters, } \\
\text { regulators, sulfur } \\
\text { metabolism }\end{array}$ & $\begin{array}{l}\text { Energy metabolism, } \\
\text { virulence, } \\
\text { adaptation }\end{array}$ & 0 \\
\hline Cy22 & 5992000.6012890 & & nocyr_5383 & nocyr_5404 & 20.9 & 21 & Enzymes & Unknown & 0 \\
\hline
\end{tabular}

end could be detected for RGP-Cy2 (R), -Cy3 (L), -Cy4 (R), -Cy7 (R), -Cy11 (R), -Cy17 (R), -Cy18 (L), -Cy19 (R), -Cy20 $(\mathrm{L}),-\mathrm{Cy} 21(\mathrm{~L})$, and -Cy22 (R) among all the strains tested. The $L$ and $R$ ends of the RGP-Cy5, Cy6, Cy10, and Cy11 were detected among the "GUH-2 complex", while only RGP-Cy1 (L), -Cy3 (R), -Cy7 (L), -Cy11 (L), -Cy13 (L),
-Cy20 (R), -Cy21 (R) could be detected among the "GUH-2 complex" (data not shown). It is noteworthy that an attempt was made at comparing this RGP classification with phylogenetic relationships inferred from $16 \mathrm{~S}$ rDNA sequences. However, significant sub-groups among $N$. cyriacigeorgica could not be resolved with this marker (data not shown). 


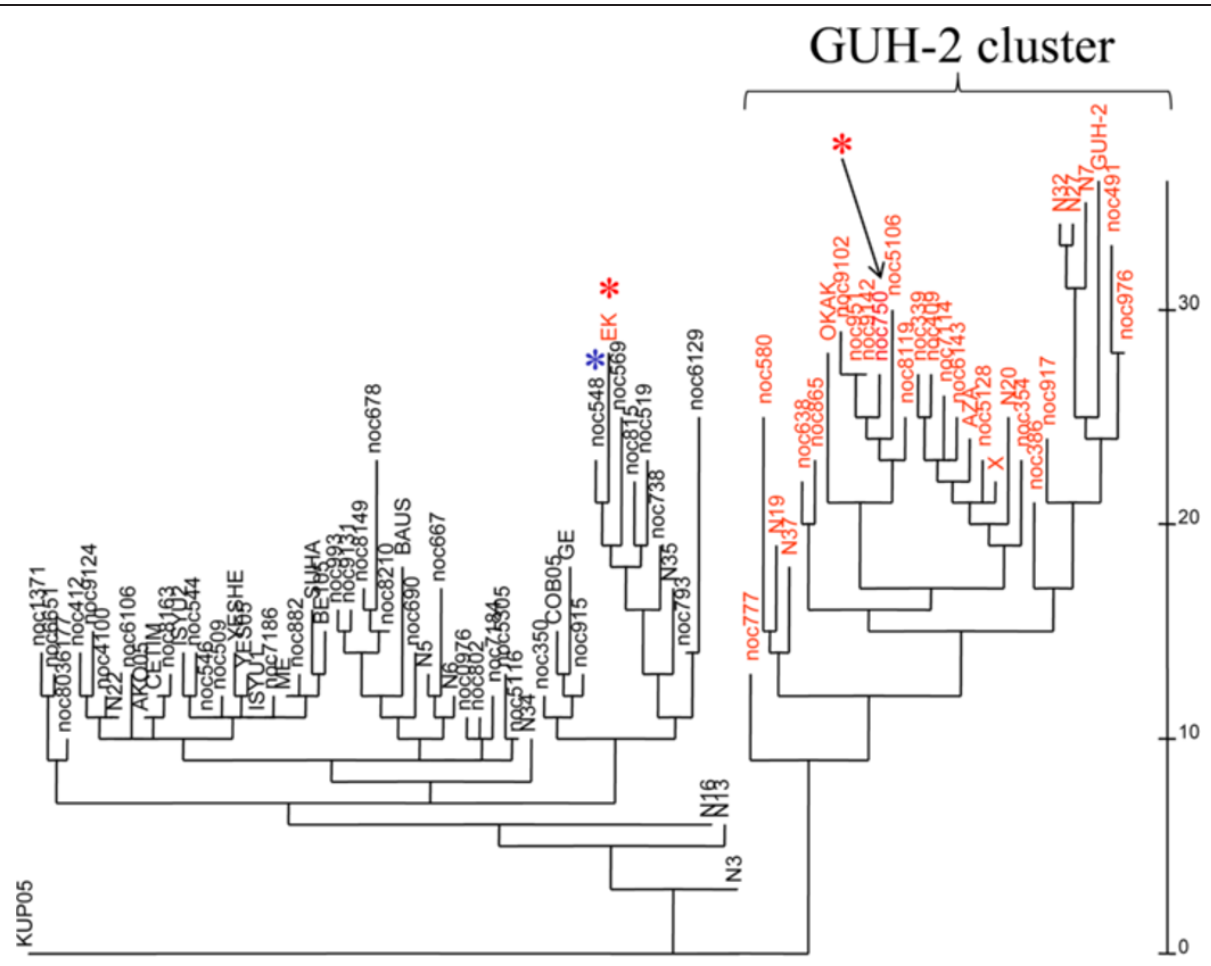

Figure 7 Cladogram illustrating the distribution of 22 RGP observed in the $N$. cyriacigeorgica GUH-2 genome among a panel of $83 \mathrm{~N}$. cyriacigeorgica strains. PCR screenings targeted three markers among each of the 22 RGP. Strains indicated in red harbored 5 or more RGP and those in black harbored less than 5 RGP. "*" indicates strains moving from one cluster to another depending on the number of markers analyzed per RGP. The scale indicates the number of changes in the RGP patterns between pairs of strains.

RGP represent gains in CDS likely to confer novel properties but CDS losses can also represent adaptations to particular habitats. $193 \mathrm{~N}$. farcinica CDS were found mISing in the $N$. cyriacigeorgica GUH-2 genome. Among these, $35 \%$ encode putative proteins of unknown function but four clusters of CDS involved in glutamine metabolism, in phenyl acid acetic degradation, in thiocyanate degradation and nitrogen metabolism, and in urease synthesis were found mISing (see Additional file 3 for further details).

\section{The particular case of insertion sequences (IS)}

On the $N$. cyriacigeorgica genome, eleven IS belonging to five different families were detected (IS3, IS21, IS200, IS256 and ISNCY). A re-evaluation of the $N$. farcinica IS genome content was performed, and led to the identification of fifteen IS belonging to five families (IS3, IS5, IS200, IS481, and IS630) (Additional file 8). Only ISNfa14 and ISNcy8 of the IS3 family were found located in the same DNA site of these two actinobacteria. These IS share $86 \%$ DNA identity. $N$ cyriacigeorgica genome showed DNA signatures of two Tn3 transposons but one appeared to be truncated. $N$. farcinica genome also showed the presence of a $\operatorname{Tn} 3$ in which the transposase CDS shares $85 \%$ DNA identity with the one of $N$. cyriacigeorgica transposon 2. Inverted repeats (IR) and/or direct repeats (DR) were identified for five $N$. cyriacigeorgica IS and for three $N$. farcinica IS. ISNcy2 copies (with DNA identities going from 82 to 100\%) in $N$. cyriacigeorgica genome were found related to IS987 (75\% DNA identity) which was only previously detected in the $M$. tuberculosis and $M$. bovis genomes. The $N$. cyriacigeorgica GUH-2 genome harbors the ISNcy1 in four copies with one being partial and three being identical. Three IS were found in several copies in $N$. farcinica. ISNfa1 of the IS481 family was found in two copies with $99 \%$ DNA identity; ISNfa2 of the IS5 family in eight copies with identities going from $72 \%$ to $99 \%$; and ISNfap1, a partial element of the Tn3 family, having $85 \%$ DNA identity with a copy found on $N$. farcinica larger plasmid.

The distribution of six of these IS/Tn elements was investigated among a sub-set $(\mathrm{n}=18)$ of the $N$. cyriacigeorgica strains screened in the RGP section, a set $(n=11)$ of $N$. farcinica strains, and a panel of Nocardia species type strains $(\mathrm{n}=12)$ (Additional file 9). Only ISNcy4 (Tn3) and ISNfa2 (IS427 of the IS5 family) were detected among both $N$. cyriacigeorgica and $N$. farcinica but their prevalence was quite different from one species to another. In fact, both of these elements were more prevalent among the panel of strains of $N$. farcinica than $N$. cyriacigeorgica strains selected for this study. ISNcy2 (IS51 of the IS3 family) was 
the most prevalent among $N$. cyriacigeorgica but was absent from the $N$. farcinica strains tested. This ISNcy2 was also found among the $N$. otitidiscaviarum type strain. ISNcy3 was not detected in $N$. farcinica. Its prevalence was higher among the GUH-2 complex of $N$. cyriacigeorgica (which was defined according to the distribution of RGP in the section above). A similar situation was observed for ISNcy4. ISNcy2 and ISNcy3 were not detected among the Nocardia type strains that were selected for this analysis, suggesting a distribution restricted to $N$. cyriacigeorgica. ISNfa1 and ISNfa5 were not detected in $N$. cyriacigeorgica and appeared to be restricted to N. farcinica strain 10152 . ISNcy4 (Tn3) and ISNfa1 (IS481) share 89\% identity, and were detected among several strains of $N$. farcinica and $N$. cyriacigeorgica. A cladogram was built from this IS distribution pattern analysis and was found to match the RGP distribution patterns (data not shown).

\section{Virulence-related functions}

Several genes were previously found involved in virulence among Actinobacteria. Blast and keyword-based searches allowed identification of some of these CDS among the $N$. cyriacigeorgica genome. Six complete mce (mammalian cell entry) loci containing $y r b E$ and mas (mce associated) CDS were found. Analysis of CDS encoding cell wall components involved in virulence revealed $85-\mathrm{kDa}$ antigens family proteins (4 CDS), lipoproteins (19 CDS) and PE_PGRS/PPE family proteins (5 CDS). Two superoxide dismutase CDS (sod) and three catalase ones were also identified. Nitrate reductase CDS (narBGHIJKY, and nirBD), trehalose 6,6'-dimycolate transferase (one CDS) and RuBisCO (2 CDS) were detected and found to be clustered.

Extracellular enzymes were identified by searching for particular domains including a peptide leader and low number of transmembrane domains. The inferred secretome of $N$. cyriacigeorgica GUH-2 was compared with the ones of other Actinobacteria, and showed a majority $(70 \%)$ of putative proteins of unknown function, several proteases, lipases as well as a transcriptional regulator and members of the mce genes (Additional file 10 shows these exported CDS in more detail). Comparison of the putative proteins of the $N$. cyriacigeorgica and $N$. farcinica secretomes showed high identities (between 81 to $86 \%$ ), much higher than those observed with other Actinobacteria (60\%).

The N. cyriacigeorgica GUH-2 genome shows a good potential for the synthesis of a number of metabolites that could be antimicrobials or proteasome inhibitors. Seven CDS were predicted to encode polyketides synthases (PKS), and 17 CDS were predicted to encode NRPS (nonribosomal peptide synthetases). Among $N$. farcinica, 4 PKS and 15 NRPSs were found including the cluster of CDS previously described as producing a lipid-soluble ironbinding nocobactin. A cluster of nine CDSs similar to the coelibactine synthesis cluster in Streptomyces coelicolor was also identified in $N$. cyriacigeorgica GUH-2's genome. NOCYR_4800 was found to be the longest CDS of the genome (43689 pb) and likely to encode a NRPS with twelve modules. Two other NRPS operons containing two and three CDS could synthesize molecules containing thirteen and eleven building blocks, respectively. A 2-amino-9,10 epoxi-8-oxodecanoic acid was predicted for NOCYR_0751. This putative acid shows a structure similar to the epoxomicin proteasome inhibitor (epoxy group) but chemical assays will be required to confirm its synthesis. Other softwares did not find this structure.

It is noteworthy that a correlation was observed between the isoelectric point and the molecular weight of the inferred proteins of the $N$. cyriacigeorgica GUH-2 genome. The largest proteins of the genome, constituted mainly by NRPS and PKS, showed similar isoelectric points, suggesting a compartmentalization inside the cytosol that could allow a coordinated functioning of these enzymes (Figure 8).

\section{Genetic potentials and metabolic profiling}

Phenotypic microarray datasets including antibiotic resistances were compared with the functional predictions made from the annotated $N$. cyriacigeorgica GUH-2 genome. Phenotypic profiling was performed by testing $N$. cyriacigeorgica ability to grow with various carbon and nitrogen sources. KEGG pathways were used to find the CDS involved in these pathways but also as a reference for explaining an absence of growth under certain conditions. (Additional file 11). Only seven amino acids (L-asparagine, L-aspartic acid, L-cysteine, L-glutamic acid, L-glutamine, L-histidine, L-lyxose, L-tyrosine) could be used as carbon and/or nitrogen sources. The absence of some CDS previously shown in other bacteria to be involved in the substrate catabolism or transport could explain several negative results. However, the lack of growth with Laspartic acid, L-lysine and L-proline could not be explained by the absence of particular CDS.

The osmoadaptation capacity N. cyriacigeorgica GUH-2 was also tested and found to be high under high salt concentrations. This property could involve CDS encoding transport proteins and/or osmoprotectants like ectoine and betaine (Additional file 11). Growth tests were also performed under various $\mathrm{pH}$. N. cyriacigeorgica GUH-2 was shown to grow at $\mathrm{pH} 7$ and 8 but not $\mathrm{pH}$ 5.2. This dataset was completed by an analysis of the antibiotics resistance pattern of $N$. cyriacigeorgica GUH-2 using plate assays. N. cyriacigeorgica GUH-2 was confirmed to have a type VI pattern with resistance against several sulfonamides, aminoglycosides, tetracyclines and penicillins. It was found sensitive to cefamandole, cefotaxime, amikacin, and imipenem. Many CDS likely playing part in these antibiotic resistances were found in the $N$. cyriacigeorgica 


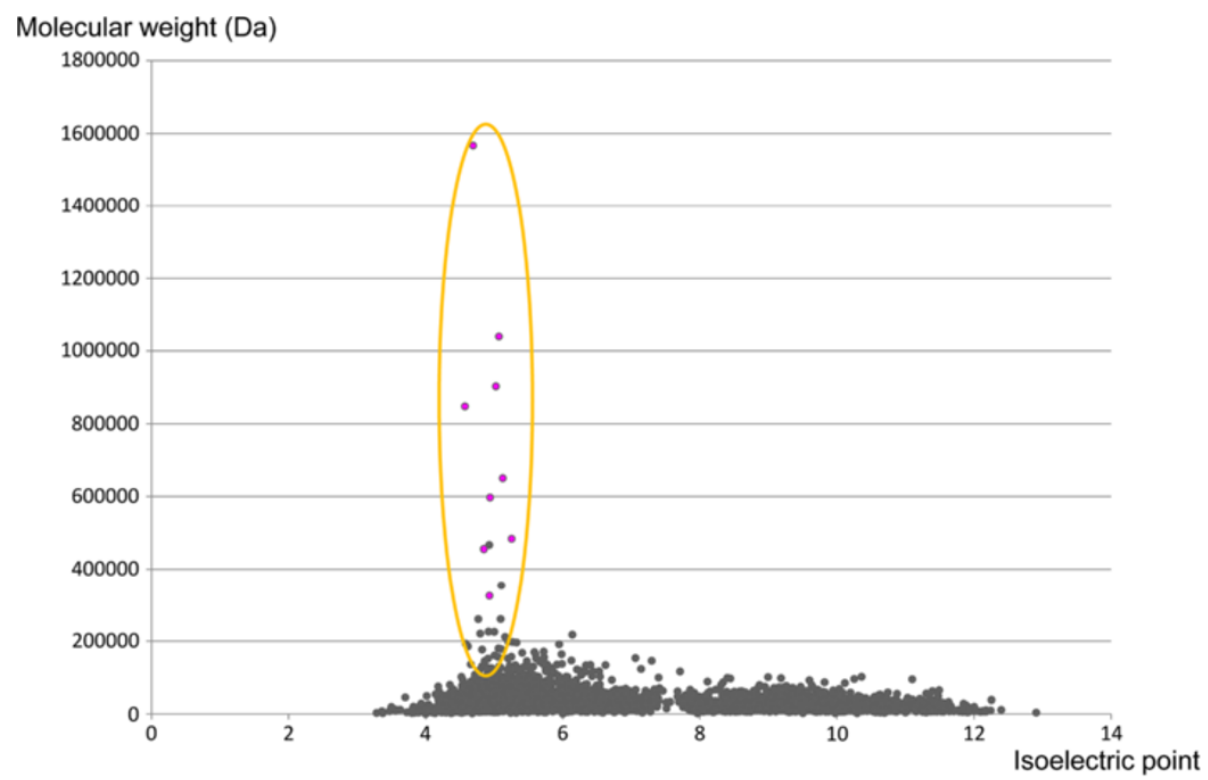

Figure 8 Relation between isoelectric point (x-axis) and molecular weight (y-axis) of $N$. cyriacigeorgica proteins.

genome. A mutation in the gyrase A gene that can prevent ciprofloxacin binding, was detected. Several CDS were inferred as encoding $\beta$-lactamases in the GUH-2 genome, and could be responsible for the observed ampicillin, carbenicillin, oxacillin and penicillin resistances. The paromomycin and tobramycin resistances observed can be due to the expression of one of the three aminoglycoside phosphotransferases detected by the annotation process. Macrolide resistances could involve one of the six ribosomal RNA methyltransferases encoded by the GUH-2 genome. These may cause a decrease in the affinity of macrolides for the $50 \mathrm{~S}$ ribosomal unit. The fourteen drug or multidrug efflux transporters encoded by this genome could be involved in tetracycline, penimepicycline, polymyxin B, paromomycin, D,L-serine hydroxamate, sisomicin, sulfamethazine, novobiocin and/or sulfadiazine resistance (data not shown) by extruding these molecules.

\section{Discussion}

Nocardia cyriacigeorgica is an opportunistic pathogen causing many infections including deadly brain abscesses and granulomatous diseases among immune-compromised and healthy individuals. However, the bacterial properties involved in these infections are poorly understood. Here, the content, organization, plasticity, and functional potentialities of $N$. cyriacigeorgica GUH-2 full genome sequence are presented. A particular attention was paid to the analysis of CDS likely involved in virulence including antibiotic resistances. These analyses led to the identification of several RGP and IS elements that were then tracked among a panel of $N$. cyriacigeorgica strains.
These screenings revealed an important ability of this species at acquiring DNA by horizontal transfer events.

\section{Virulence determinants}

The $N$. cyriacigeorgica GUH-2 genome analyses revealed several genetic determinants related to virulence. Some of these are part of RGP but some were also part of the Nocardia or Actinobacteria pangenomes inferred from comparisons with the full genome sequence of $N$. farcinica and other Actinobacteria. The genome of $N$. cyriacigeorgica harbors virulence-related CDS such as Mce coding genes described as important virulence factors of $M$. tuberculosis [30]. Mce can act as transmembrane transporters favoring macrophage invasion [31]. These CDSs are organized in operons containing two $y r b E$ CDS followed by six mce CDS and sometimes two $m c e$ associated (mas) CDSs. There are six copies of the complete mce operon in $N$. cyriacigeorgica and $N$. farcinica whereas four copies are identified in M. tuberculosis genome. Although, the importance of these CDSs in $M$. tuberculosis virulence has been shown [32], their detection in multicopies among $M$. smegmatis and $R$. jostii (six and four clusters, respectively) suggests a function not limited to cell entry [33]. Having access to the $N$. cyriacigeorgica mce CDS will allow transcriptomics experiments to identify the mce CDSs which are turned on during host cell colonization.

Other $N$. cyriacigeorgica CDSs besides mce have been described as important in the infection and cell invasion processes. In particular, the expression of superoxide dismutase and catalase CDS were observed during $N$. 
cyriacigeorgica macrophage invasion and were suggested to be involved in resistance towards oxidative stresses [34]. Two sod and three kat CDS were found in the $N$. cyriacigeorgica genome. The katA CDS is harbored by the RGP-Cy3 but the other two kat CDSs are found on conserved genomic regions. Other CDSs can also have a complementary action during Nocardia growth in macrophages. The trehalose 6,6'-dimycolate transferase CDS was found related to the $85-\mathrm{kDa}$ antigen family protein [35] that can promote $M$. tuberculosis survival in macrophages by decreasing both phagosomal acidification and phagolysosomal fusion [36,37]. An encoded $N$. cyriacigeorgica hemolysin which can disrupt the phagolysosome membrane was also annotated and could also favor survival in macrophages $[38,39]$. The isocitrate lyase CDS observed on RGP-Cy15 could also be part of the $N$. cyriacigeorgica macrophage invasion process by preventing host cell apoptosis as observed in M. tuberculosis [40]. An intracellular pathogen not only requires defense mechanisms against macrophage antibacterial processes but also needs to survive under low oxygen pressure such as the one observed in poorly irrigated tissue of the mammal body [41]. CDS involved in such processes can thus also be defined as virulence-related determinants. $N$. cyriacigeorgica contains denitrification CDSs (narBGHIJKY, and nirBD) of which five are harbored by RGP-Cy14. N. cyriacigeorgica was initially described as an obligate aerobe but presence denitrifying CDS suggests an ability to grow under anaerobic conditions. However, the conditions allowing growth under low oxygen pressure remain to be defined.

Several metabolites, proteins, enzymes and lipids which are not directly involved in colonization but are at the frontline during host infection can also play a role in virulence such as cell wall constituents and some extracellular enzymes/metabolites. The cell wall is a protection for bacteria but can also be a target for the immune system. For example, PE/PPE serine $\alpha / \beta$ hydrolases membrane proteins are important in Mycobacterium pathogenic species to avoid detection or killing during their intracellular life in a variety of host cells $[42,43]$. However, their low number in $N$. cyriacigeorgica and $N$. farcinica could be an effect of their opportunistic status (environmental cycle) and could explain their poor ability to escape the immune system of a healthy host. Nocardia can also secrete several enzymes that may interfere with the host cell metabolism [25], in particular SODs [44], lipases [45] and proteases [46]. CDSs encoding such enzymes were observed in the $N$. cyriacigeorgica GUH-2 genome. Eight CDSs were found encoding extracellular lipases and 10 encoding extracellular proteases. Nevertheless, much more CDSs encoding putative secreted enzymes have been reported in Mycobacterium such as the ESAT (early secreted antigenic target) proteins [47]. N. cyriacigeorgica GUH-2 genome harbored three ESAT CDSs.
Another major group of secreted molecules that can play a role in virulence are siderophores. Nocardia strains can produce several siderophores like formobactin [48], amamistatin [49], brasilibactin [50], asterobactin [51] and nocobactin [52]. Nocobactin synthesis was previously found encoded by two genetic clusters, with cluster I positioned $195 \mathrm{~kb}$ from cluster II. The $N$. cyriacigeorgica GUH-2 genome showed a cluster I organization different from the one observed in the $N$. farcinica genome. The gene $n b t H$ is absent in the $N$. cyriacigeorgica GUH-2 genome and replaced by a putative formyltransferase CDS, which could play a role in preventing the transfer of an acyl chain to the $\varepsilon$-amino group of lysine. The $N$. cyriacigeorgica GUH-2 cluster II shows high identities with the one of $N$. farcinica but additional CDS encoding NRPS and an exported protein of unknown function were recorded. The NRPS CDSs could play a role in the synthesis of this siderophore and change some of its properties. A coelibactin-like siderophore is also likely to be produced by $N$. cyriacigeorgica GUH-2. A conserved synteny was observed with $S$. coelicolor CDS involved in its synthesis except for a supplementary CDS encoding a cytochrome P450 protein.

These Actinobacteria are also known to synthesize other extracellular metabolites through NRPS and PKSrelated processes. The $N$. cyriacigeorgica GUH-2 genome was found to encode 12 PKS-related CDSs and 22 NRPS-related ones. It is considered that the substance produced by $N$. cyriacigeorgica GUH-2 which can cause brain damages by inducing apoptosis and a dopamine depletion would be encoded by a NRPS or PKS $[25,26]$. Here, a putative metabolite produced by an operon of three NRPSs could be predicted to have a structure similar to the one of epoxomicin [53]. Such molecules have epoxy groups that could inhibit the functioning of proteasomes. NRPS and PKS are also involved in the synthesis of antibiotics, and could have been involved in the synthesis of transvalencin Z [54], DA-7218 [55], and nocardithiocin [56]. However, the molecules produced by these synthases are often difficult to obtain in large quantities and are hard to purify from culture filtrates without genetic manipulations.

\section{Phenotypic and genomic plasticities}

Metabolic profilings showed $N$. cyriacigeorgica GUH-2 abilities at growing on a variety of substrates. In most cases, the CDSs involved in transport and catabolism of these substrates were found in the genome. However, some metabolic activities inferred from the genome could not be confirmed by growth tests e. g. the growth on L-glycine, L-lysine, and L-proline. Interestingly, CDSs involved in L-lysine and L-proline catabolism were detected outside RGP but showed a codon adaption index (CAI) below the average (data not shown). This 
codon bias could be related to the low expression of these CDSs, and might be the consequence of a recent acquisition. An overexpression of these genes by genetic manipulations of $N$. cyriacigeorgica GUH-2 would be needed to test this hypothesis. Low CAI was also observed for CDSs identified when in vivo activity was assessed. In this case the presence of multiple CDS (with both high and low CAI values) assigned to this function probably allowed a sufficient gene expression level to observe the expected phenotype.

A high turnover of RGP appears to occur among $N$. cyriacigeorgica. $N$. cyriacigeorgica GUH-2 closest RGP profile among a collection of 83 strains showed the absence of 7 RGP and the conservation of 15 ones. RGP profilings thus suggest a good $N$. cyriacigeorgica competence towards DNA acquisition but the mechanisms involved remain to be determined. RGP-Cy8 appeared to have been recently acquired by $N$. cyriacigeorgica GUH-2, and was found to harbor all the trademarks of mobile genomic islands. This RGP could be a good candidate for the design of a DNA cloning vector [57]. No RGP was found related to prophage-like elements. This is different from the situation observed in corynebacterial and mycobacterial pathogenic genomes, where such prophage-like DNA contains virulence genes [28].

A high turnover of IS elements was also observed among Nocardia. However, while several IS were found among the $N$. cyriacigeorgica $(\mathrm{n}=16)$ and $N$. farcinica $(\mathrm{n}=26)$ genomes in this work, previous studies had only identified two IS among the Nocardia: ISNfa1 (N. farcinica) [41] and IS204 (N. mexicana) [58]. These low numbers suggested Nocardia strains to have a poor propensity at acquiring exogenous DNA which are often acting as IS shuttles. Here, we clearly demonstrate the opposite. The Nocardia genomes were found to be rich in IS elements and diversity. IS of eight families were recorded suggesting a high genomic tolerance towards these elements and a frequent acquisition by these Actinobacteria. However, about $45 \%$ of the observed IS did not show IR and DR. A lack of such sequences could be indicative of a loss of transposition autonomy or of selective pressures leading to their fixation at a particular site because of functional benefits. This would need to be further investigated. IS were found involved in the emergence of pathogenic clones by reducing genome size [59]. There are also some reports showing a good match between the presence of a particular IS and infra-specific diversifications e.g. [60]. Distribution analysis also showed species-specific IS elements among the Nocardia e.g. ISNcy2 and ISNfa2 being restricted to $N$. cyriacigeorgica and $N$. farcinica, respectively. Furthermore, ISNcy3 was restricted to the $N$. cyriacigeorgica GUH-2 complex, and ISNfa5 of two particular clones of $N$. farcinica. These data support the hypothesis of a good match between IS acquisitions and infra-specific diversifications. Interestingly, distribution analysis indicated similar RGP/IS repartition profiles among $N$. cyriacigeorgica strains. These profiles divided $N$. cyriacigeorgica into two clusters: (1) those with patterns similar to strain GUH-2, and (2) those with patterns similar to the type strain. These similarities in the evolutionary patterns of these elements suggest a strong association. RGP could have been the genetic shuttles for some of these IS elements. In fact, even though most IS elements were found distributed over the genome without particular insertion site preferences, some e.g. ISNcy5, ISNcy2-b, ISNCy4, \& ISNcy1-a4, were found harbored by RGP. For example, ISNcy2-b could have been acquired with RGP-Cy13. This IS is significantly different from the other ISNcy2-related copies found in the $N$. cyriacigeorgica GUH-2 genome, and has DNA signatures only detected on the copy found on this RGP. Interestingly, a division of $N$. cyriacigeorgica into two phylogenetic clusters was inferred by McTaggart et al. [13]. However, the strain collection of this latter study was different from the one of this work. It would be interesting to apply McTaggart et al. [13] approach to see if the IS/GI sub-groups would match such phylogenetic clusters. These analyses would add further support for the role of IS/GI elements in bacterial diversification and speciation. No relation between the habitat nor the geographical origin of the strains used in our study and the observed RGP/IS patterns could be inferred.

It is noteworthy that ISNcy2 was found similar to IS987 of $M$. tuberculosis and M. bovis, and was not detected in other sequenced bacterial genomes. In order to relate IS divergences to the evolution of Actinobacteria, the two orthologous IS alleles named ISNfa14 and ISNcy8, found in $N$. farcinica and N. cyriacigeorgica, respectively, were used as molecular clocks. These IS have $86 \%$ identity. Using this value, one can consider ISNcy2 and IS987 diversification ( $75 \%$ identity) to be more ancient. PCR screenings showed ISNcy2 to be broadly distributed among $N$. cyriacigeorgica whereas it was totally absent from $N$. farcinica and other Nocardia type strains. This would suggest an acquisition by $N$. cyriacigeorgica at the moment of its differentiation.

\section{Outdoor-related functional ISues}

Although a significant number of virulence genes were identified, with some being harbored by RGP, the main drivers of $N$. cyriacigeorgica evolution appear to be related to its environmental cycle outside mammalian hosts. Nocardioses are without a doubt of environmental origin, and infections are mainly the consequence of exposure to soil or water sources of these opportunistic pathogens. Indeed, several characteristics of the $N$. cyriacigeorgica GUH-2 genome reflect the environmental origin of this species. For instance, $N$. cyriacigeorgica 
genome size is in the range of what is expected for saprophytic bacteria (from $6 \mathrm{Mb}$ to $11 \mathrm{Mb}$ ). This higher size range is related to the selection of CDS likely improving metabolic potentials and regulatory processes, and allowing growth under a wider range of environmental constraints [61]. These observations were further supported by COG analyses which showed Nocardia genomes to share a configuration similar to the ones observed among nonpathogenic Actinobacteria. Interestingly, both Nocardia genomes have a higher proportion of $\mathrm{K}$ and T COG CDS. These COGs encode DNA and proteins with regulatory functions, suggesting an ongoing evolution towards regulatory fine tunings of their genetic potentials that likely lead to expression patterns favoring growth under more diverse conditions.

\section{Conclusions}

On one hand, primary pathogens are subjected to evolutionary forces driven by the host defense responses which can lead to a specialization for certain hosts and a genome size reduction as observed for B. mallei [62]. On the other hand, opportunistic pathogens are exposed to a multitude of environmental constraints that can favor an increased tolerance towards the acquisition of foreign DNA and the selection of novel metabolic properties. The $N$. cyriacigeorgica GUH-2 genome is a clear reflection of these latter trends. The $N$. cyriacigeorgica GUH-2 genome shows a great plasticity as shown from its RGP and IS patterns, which are strain-specific and appeared to have recently evolved. In fact, this genome appears to be undergoing important genetic rearrangements, and, most surprisingly, to frequently acquire novel DNA fragments. So far, Nocardia spp. were thought to have a low mating frequency, not acquiring much novel DNA from their neighbors. This work shows a completely opposite trend. N. cyriacigeorgica GUH-2 is clearly competent towards DNA or sexually active. This property can favor the gain of novel functions, and lead to major changes in niche preference from one strain to another such as differences in the colonization of certain human tISues.

\section{Methods}

\section{Mice experimentations}

Female BALB/c mice (pathogen-free) of 18-20 g (approximately 8 weeks old) were maintained by the "Institut Claude Bourgelat" (VetAgroSup, Marcy l'Etoile, France) in accordance with protocols approved by the board of ethics for animal experimentations.

$N$. cyriacigeorgica GUH-2 strain was grown in brainheart infusion broth (BHI-P) to mid-log phase at $37^{\circ} \mathrm{C}$ with mild rotational agitation $(150 \mathrm{rpm})$. The broth was centrifuged at low speed ( $55 \mathrm{~g}$ ) to pellet bacterial clumps, and cell concentration was adjusted at approximately
$3.5 \times 10^{6} \mathrm{CFU} / \mathrm{ml}$. A $0.1 \mathrm{ml}$ of this suspension was injected intravenously (IV) through the lateral tail vein into each mouse, as described in details by Kohbata and Beaman [18]. Each mouse received approximately $3.5 \times$ $10^{5}$ CFU.

\section{Genome sequencing and assembly}

The genome sequence of $N$. cyriacigeorgica GUH-2 is publicly available at http://www.genoscope.cns.fr/agc/ mage [63]. Sequences and annotations data have been deposited at the EMBL database (http://www.ebi.ac.uk/ ena/) and given the accession number FO082843.1.

\section{Genome annotation and analysis Synteny group computation}

Sequence data for comparative analyses were obtained from the NCBI database (RefSeq section). Putative orthologies were defined as gene pairs satisfying either the $\mathrm{BBH}$ criterion or an alignment threshold (at least $40 \%$ sequence identity over at least $80 \%$ of the length of the smallest protein) [64]. These relationships were subsequently used to search for synteny groups (i.e. conservation of the chromosomal co-localization between pairs of orthologous genes from different genomes) among several bacterial genomes using an algorithm based on an exact graph-theoretical approach [65]. These results were used to draw a LinePlot using the MaGe MicroScope platform (https://www.genoscope.cns. fr/agc/microscope/home/index.php).

\section{Detection of regions of genomic plasticity}

The RGPfinder tool of the MicroScope annotation platform was used to identify Regions of Genomic Plasticity (RGP) in the whole genome sequence of $N$. cyriacigeorgica by using the $N$. farcinica sequence as a reference. RGP are defined as regions of at least $5 \mathrm{~kb}$ that are mISing in at least one of the genomes that are compared. This definition makes no assumption about the evolutionary origin or genetic basis of these variable chromosomal segments. RGPfinder searches for synteny breaks between a target genome and a set of closely related bacteria (generally other strains). It also provides information about composition abnormalities (\%G $+\mathrm{C}$ deviation, Codon Adaptation Index) of these regions, and of their flanking sequences such as tRNA genes, IS and repeats, which are common features of RGP. Moreover, the tool integrates the results of Alien Hunter [66] and SIRGP-HMM [67], two methods that analyses compositional biases to detect atypical sequences (i.e. sequences potentially acquired by horizontal gene transfer). The whole genome of the N. cyriacigeorgica and $N$. farcinica were also aligned using ACT, a program for comparing two or more DNA sequences [68]. A region of five or more CDS and of more than $5 \mathrm{~kb}$ not retrieved in $N$. farcinica was also considered a RGP. 


\section{Global comparative study}

We compared genomes of $A$. mediterranei (CP002896.1), C. diphtheriae (BX248353), C. glutamicum (BA000036), M. tuberculosis (AL123456), M. smegmatis (CP000480), N. farcinica (AP006618), N. cyriacigeorgica (FO082843), $R$. equi (ADNW00000000) and $R$. jostii (CP000431) using various graphic tools implemented on the MaGe Microscope platform and ACT. The phylogenetic tree of life of the Actinobacteria was built from a MLSA data set on the basis of [13]. We used Clusters of Orthologous Groups (COGs) automatic annotation and correspondence analysis (CA) with $\mathrm{R}$ software (http://www.R-project.org) [69] to graphically infer global trends between the above genomes. Nocardia IS families were identified using BLAST analyses against an IS database at https:// www-is.biotoul.fr/.

Deleted CDS from the $N$. cyriacigeorgica GUH-2 genome were identified by searching CDS in common with $N$. farcinica, $R$. equi and $R$. jostii and looking for those absent from $N$. cyriacigeorgica genome using the Phyloprofiles exploration tool of MaGe. Only CDS presenting more than $40 \%$ amino-acid identities over $80 \%$ of the length of the shortest sequence were considered. Duplicated genes were detected in the $N$. cyriacigeorgica GUH-2 genome with the same tool, $N$. cyriacigeorgica genome was compared against itself and CDS presenting more than $70 \%$ predicted amino-acid identities were selected.

\section{Distribution of IS and selected RGP among a set of Nocardia strains}

Rapid DNA extractions were performed on the suspension of 10 to 20 colonies of Nocardia cells in $100 \mu \mathrm{L}$ of sterile water. The mixture was heated to $55^{\circ} \mathrm{C}$ for $15 \mathrm{~min}$, and 5 units of achromopeptidase (Wako chemicals, Richmond VA) were added before incubating the suspension at $70^{\circ} \mathrm{C}$ for $15 \mathrm{~min}$. Cells were centrifuged and the supernatant containing DNA was kept for further analyses. PCR screenings were designed to investigate the distribution of all selected RGP reported in this work, and for a set of selected IS elements. Primers were defined using primerselect (DNASTAR), and are indicated in the Additional file 12. All PCR reactions were performed in a final volume of $25 \mu \mathrm{L}$, containing $2.5 \mu \mathrm{L}$ of each primers at $10 \mu \mathrm{M}, 2.5 \mu \mathrm{L}$ of $10 \mathrm{X}$ PCR buffer, $0.75 \mu \mathrm{L}$ of $\mathrm{MgCl}_{2} 50 \mathrm{mM}, 0.25 \mu \mathrm{L}$ of $10 \mathrm{mM}$ DNTPs, $5 \%$ DMSO w.vol ${ }^{-1}$, and $1 \mu \mathrm{L}$ of the extracted DNA solution. PCR cycle is: $95^{\circ} \mathrm{C} 300 \mathrm{sec}, 95^{\circ} \mathrm{C} 30 \mathrm{sec}$, annealing temperature depending on primers used, $72^{\circ} \mathrm{C}$ from $30 \mathrm{sec}$ to $90 \mathrm{sec}(\times 35)$ and $72^{\circ} \mathrm{C} 300 \mathrm{sec}$. PCR products were visualized by electrophoresis using $1 \%$ agarose gels, and staining with ethidium bromide.

\section{Identification of the secretome}

Protein secretion in Gram-positive bacteria occurs mainly through general secretory $(\mathrm{Sec})$ and twin arginine translocation (Tat) pathways, and to a lesser extent by ABC (ATP-binding cassette) type transporters. There are also minor pathways such as the Early Secreted Antigen Target (ESAT-6) machinery described in Mycobacterium $[70,71]$. The secretome was analyzed for pathogenic and non pathogenic Actinobacteria using SignalP and TMhmm [72] as well as PSORTb [73]. These bioinformatics tools can detect trans-membrane domains [74]. None of these tools is error-free but their combined use yields a set of proteins that were previously shown, in most cases, to be secreted proteins [72,75].

\section{Metabolic profiling}

N. cyriacigeorgica GUH-2 strain was grown for three days at $37^{\circ} \mathrm{C}$ on Middlebrook $7 \mathrm{H} 10$ (supplemented with $0.5 \%$ glycerol and 1\% Middlebrook OADC enrichment) agar plates. $N$. cyriacigeorgica GUH-2 was inoculated to $20 \mathrm{~mL}$ of IF-0a GN/GP (Biolog Inc, Hayward CA, USA) and homogenized to obtain a $81 \%$ transmittance solution, free of bacterial clumps. This suspension $(880 \mu \mathrm{L})$ was added to $10 \mathrm{~mL}$ of IF-0a GN/GP (Biolog Inc) supplemented with $1 \mathrm{~mL}$ of solution specific of each Omnilog plate. Dye mix $(120 \mu \mathrm{L}) \mathrm{F}$ or $\mathrm{H}$ (depending on the Omnilog plate use) was added. This mix $(100 \mu \mathrm{L})$ was added to each well of the selected Omnilog plates, and the plates were incubated. Cellular respiration was measured by monitoring formation of dark blue tetrazolium crystals over a $72 \mathrm{~h}$ time period.

\section{Additional files}

\section{Additional file 1: Virulence-related CDS found in the $N$. cyriacigeorgica GUH-2 genome.}

Additional file 2: $N$. cyriacigeorgica GUH-2 CDS occurrence and proportion in the CMN pangenome per COG. Proportion of $N$. cyriacygeorgica CDS per COG was compared with the ones of the CMN pangenome by using the following formula: number of $\mathrm{N}$. cyriacygeorgica CDS in a COG of the CMN pangenome divided by the total number of $N$. cyriacygeorgica CDS in this COG.

Additional file 3: Analysis of duplicated (threshold of $70 \%$ identity) lost (threshold of $40 \%$ identity), and RGP CDS of $N$. cyriacigeorgica GUH-2. Deleted CDS from the $N$. cyriacigeorgica GUH-2 genome were identified by searching CDS in common with $N$. farcinica, $R$. equi and $R$. jostii and looking for those absent from $N$. cyriacigeorgica genome using the Phyloprofiles exploration tool of MaGe. Only CDS presenting more than $40 \%$ amino-acid identities over $80 \%$ of the length of the shortest sequence were considered.

Additional file 4: Number of COGs and their relative proportion per species computed from nine Actinobacterial genomes.

Additional file 5: Correspondence Analysis of domains involved in transcription and retrieved in Am (A. mediterranei), $\mathrm{Cd}(C$. diphtheria), Cg (C. glutamicum), Mt (M. tuberculosis), Ms ( $M$. smegmatis), Nc ( $N$. cyriacigeorgica), Nf ( $N$. farcinica), Re ( $R$. equi) and $\mathrm{Rj}(\boldsymbol{R}$. jostii). Pathogenic Actinobacteria are represented in red, the nonpathogenic or saprophytic ones are in blue and Nocardia strains are in orange. Arrows show different proportions of COGs between pathogenic and non-pathogenic bacteria in the same genera. Transcription domains are as follow: ab (AbrB), ac (AraC), ar (ArsR), as (AsnC), cr (Crp), dr (DeoR), fu (Fur), gr (GntR), hr (Hx|R), ir (ICIR), li (Lacl), Ir (LuxR), ly (LysR), ma (MarR), 
me (MerR), mo (MoxR), nr (NrdR), pr (PadR), r2 (Rrf2), tf (CarD/TRCF), tr (TetR) and wb (WhiB).

Additional file 6: Number of CDS containing putative domains involved in transcription and their relative proportion per species as computed from nine Actinobacterial genomes.

Additional file 7: General features of IS identified in the $N$. cyriacigeorgica and $N$. farcinica genomes.

Additional file 8: Number of CDS associated with the secretome of Actinobacteria, and their relative proportion per species (Nc: $N$. cyriacigeorgica, Nf: N. farcinica, Rj: R. jostii, Re: R. equi, Ms: $M$. smegmatis, Mt: M. tuberculosis, Cg: C. glutamicum, Cd: C. diphtheria, Am: A. mediterranei). Each CDS was associated to a COG.

Additional file 9: PCR primers used in this study for RGP and IS distribution analyses.

Additional file 10: Regions of genomic plasticity (Cy code) detected by PCR in $83 \mathrm{~N}$. cyriacigeorgica strains. N. farcinica DNA was used as a control.

Additional file 11: Distribution of selected IS (ISNCy) among $N$. cyriacigeorgica and $N$. farcinica strains (PCR screenings); positive results are in grey.

Additional file 12: OmniLog $^{\circledR}$ metabolic profilings of $N$.

cyriacigeorgica GUH-2 cells. Functional predictions made from genome DNA sequence analyses are indicated. Utilization of various carbon and nitrogen sources, and osmolite resistances were investigated. Differences in the data sets are indicated in grey.

\section{Abbreviations}

CDS: Coding sequence; CMN: Corynebacteria, mycobacteria, and nocardia; COG: Cluster of orthologous groups; RGP: Region of genomic plasticity; IS: Insertion sequence; IR: Inverted DNA repeats; DR: Direct DNA repeats; NRPS: Non ribosomal peptide synthetase; PKS: Polyketide synthase; CAl: Codon adaptation index; MY: Million years.

\section{Competing interests}

The authors declare that they have no competing interests.

\section{Authors' contributions}

$A Z, P P, A G, P B, Y R, B C$, and $D B$ designed the study. $A Z, P P, V R N, S R, D B$ and PBe performed the experiments. $\mathrm{AZ}, \mathrm{PBe}, \mathrm{PB}, \mathrm{PN}, \mathrm{DR}, \mathrm{DV}, \mathrm{VB}, \mathrm{SM}, \mathrm{BC}$ and $\mathrm{DB}$ analyzed the data sets. $A Z, D B$ and $B C$ drafted the manuscript; $A Z, P P, P N$, $\mathrm{BC}$, and $\mathrm{DB}$ revised the manuscript and provided critical comments. All authors approved the final version of the manuscript.

\section{Acknowledgements}

Thanks are expressed to D. Abrouk and S. Bouchet for help with treatment of large data sets. Thanks are expressed to L. Loiseau and E. Bergeron of the PARMIC and EML - biological resource center that made available the Omnilog Combo system and some of the strains used in this study. Thanks to BL. Beaman (University of California, Davis, Davis, California) for help with animal experiment procedure. This project was funded by a Genoscope research program, by a CNRS MIE interdisciplinary program and a CNRS-USA exchange program. Part of this work was funded by ANR SEST (2005-2009) project 5605 and ANR Contaminants et Environnements : Métrologie, Santé, Adaptabilité, Comportements et Usages (CESA) (2012-2016) project "CABRRES". The CNRS, University Lyon 1 and VetAgroSup institutions also contributed financially to this work. A. Zoropogui received a PhD grant from Cluster Environnement of the Rhône-Alpes region. We thank F. Poly, and V. Grossi (U. Lyon 1) for assistance with the RuBisCO assays, L. Normand (U. Lyon 1) and O. Leveneur (Institut Claude Bourgelat) for assistance with the animal experimentations.

\section{Author details}

'Research group on "Bacterial Opportunistic Pathogens and Environment", Université de Lyon, Lyon, France. ${ }^{2}$ Research group on "Actinorhizal symbiosis", Université de Lyon, Lyon, France. ${ }^{3}$ Research group on "Environmental Microbiology Lyon - Biological Resource Center", UMR5557 Ecologie Microbienne, Université de Lyon, Université Lyon 1, CNRS, and
VetAgro Sup Veterinary Campus, Lyon, France. ${ }^{4}$ Commissariat à l'Energie Atomique, Genoscope 91057, Evry cedex, France.

Received: 5 October 2012 Accepted: 19 March 2013 Published: 27 April 2013

\section{References}

1. Stackebrandt E, Sproer C, Rainey FA, Burghardt J, Pauker O, Hippe H: Phylogenetic analysis of the genus Desulfotomaculum: evidence for the misclassification of Desulfotomaculum guttoideum and description of Desulfotomaculum orientis as Desulfosporosinus orientis gen. nov., comb. nov. Int J Syst Bacteriol 1997, 47:1134-1139.

2. Embley TM, Stackebrandt E: The molecular phylogeny and systematics of the actinomycetes. Annu Rev Microbiol 1994, 48:257-289.

3. Zhao GZ, Li J, Zhu WY, Klenk HP, Xu LH, Li WJ: Nocardia artemisiae sp. nov., an endophytic actinobacterium straind from surface-sterilized stem of Artemisia annua L. Int J Syst Evol Microbiol 2011, 61:2933-2937.

4. Yassin AF, Rainey FA, Steiner U: Nocardia cyriacigeorgici sp. nov. Int J Syst Evol Microbiol 2001, 51:1419-1423.

5. Conville PS, Witebsky FG: Organisms designated as Nocardia asteroides drug pattern type $\mathrm{VI}$ are members of the species Nocardia cyriacigeorgica. J Clin Microbiol 2007, 45:2257-2259.

6. Brown-Elliott BA, Brown JM, Conville PS, Wallace RJ Jr: Clinical and laboratory features of the Nocardia spp. based on current molecular taxonomy. Clin Microbiol Rev 2006, 19:259-282.

7. Lalitha P: Nocardia keratitis. Curr Opin Ophthalmol 2009, 20:318-323.

8. Elsayed S, Kealey A, Coffin CS, Read R, Megran D, Zhang K: Nocardia cyriacigeorgica septicemia. J Clin Microbiol 2006, 44:280-282.

9. Barnaud G, Deschamps C, Manceron V, Mortier E, Laurent F, Bert F, Boiron P, Vinceneux P, Branger C: Brain abscess caused by Nocardia cyriacigeorgica in a patient with human immunodeficiency virus infection. J Clin Microbiol 2005, 43:4895-4897.

10. Beaman BL, Maslan S: Effect of cyclophosphamide on experimental Nocardia asteroides infection in mice. Infect Immun 1977, 16:995-1004.

11. Schlaberg R, Huard RC, Della-Latta P: Nocardia cyriacigeorgica, an emerging pathogen in the United States. J Clin Microbio/ 2008, 46:265-273.

12. Cargill JS, Boyd GJ, Weightman NC: Nocardia cyriacigeorgica: a case of endocarditis with dISeminated soft-tISue infection. J Med Microbiol 2010, 59:224-230.

13. McTaggart LR, Richardson SE, Witkowska M, Zhang SX: Phylogeny and identification of Nocardia species on the basis of multilocus sequence analysis. J Clin Microbiol 2010, 48:4525-4533.

14. Rodriguez-Nava V, Zoropogui A, Laurent F, Blaha D, Couble A, Mouniee D, Boiron P: La nocardiose, une maladie en expansion, Nocardiosis, an expanding disease. Antibiotiques 2008, 10:115-127.

15. Khan ZU, Neil L, Chandy R, Chugh TD, Al-Sayer H, Provost F, Boiron P: Nocardia asteroides in the soil of Kuwait. Mycopathologia 1997, 137:159-163.

16. Quatrini P, Scaglione G, De Pasquale C, Riela S, Puglia AM: Isolation of Gram-positive n-alkane degraders from a hydrocarbon-contaminated Mediterranean shoreline. J Appl Microbiol 2008, 104:251-259.

17. Le TN, Mikolasch A, Awe S, Sheikhany H, Klenk HP, Schauer F: Oxidation of aliphatic, branched chain, and aromatic hydrocarbons by Nocardia cyriacigeorgica straind from oil-polluted sand samples collected in the Saudi Arabian Desert. J Basic Microbiol 2010, 50:241-253.

18. Kohbata S, Beaman BL: L-dopa-responsive movement disorder caused by Nocardia asteroides localized in the brains of mice. Infect Immun 1991 59:181-191.

19. Beaman BL, Canfield D, Anderson J, Pate B, Calne D: Site-specific invasion of the basal ganglia by Nocardia asteroides GUH-2. Med Microbiol Immunol 2000, 188:161-168.

20. Hubble JP, Cao T, Kjelstrom JA, Koller WC, Beaman BL: Nocardia species as an etiologic agent in Parkinson's disease: serological testing in a casecontrol study. J Clin Microbiol 1995, 33:2768-2769.

21. Salama M, Arias-Carrion O: Natural toxins implicated in the development of Parkinson's disease. Ther Adv Neurol Disord 2011, 4:361-373.

22. Ogata SA, Beaman BL: Site-specific growth of Nocardia asteroides in the murine brain. Infect Immun 1992, 60:3262-3267.

23. Beaman BL, Tam S: An unusual murine behavior following infection with log-phase Nocardia asteroides type 6 strain GUH-2 (Nocardia cyriacigeorgica GUH-2). Microbes Infect 2008, 10:840-843. 
24. Tam S, Barry DP, Beaman L, Beaman BL: Neuroinvasive Nocardia asteroides GUH-2 induces apoptosis in the substantia nigra in vivo and dopaminergic cells in vitro. Exp Neurol 2002, 177:453-460.

25. Loeffler DA, Camp DM, Qu S, Beaman BL, LeWitt PA: Characterization of dopamine-depleting activity of Nocardia asteroides strain GUH-2 culture filtrate on PC12 cells. Microb Pathog 2004, 37:73-85.

26. Barry DP, Beaman BL: Nocardia asteroides strain GUH-2 induces proteasome inhibition and apoptotic death of cultured cells. Res Microbiol 2007, 158:86-96.

27. Darwin KH, Ehrt S, Gutierrez-Ramos JC, Weich N, Nathan CF: The proteasome of Mycobacterium tuberculosis is required for resistance to nitric oxide. Science 2003, 302:1963-1966.

28. Ventura M, Canchaya C, Tauch A, Chandra G, Fitzgerald GF, Chater KF, van Sinderen D: Genomics of Actinobacteria: tracing the evolutionary history of an ancient phylum. Microbiol Mol Biol Rev 2007, 71:495-548.

29. Tekaia F, Lazcano A, Dujon B: The genomic tree as revealed from whole proteome comparisons. Genome Res 1999, 9:550-557.

30. Arruda S, Bomfim G, Knights R, Huima-Byron T, Riley LW: Cloning of an M. tuberculosis DNA fragment associated with entry and survival inside cells. Science 1993, 261:1454-1457.

31. Mohn WW, van der Geize R, Stewart GR, Okamoto S, Liu J, Dijkhuizen L, Eltis LD: The actinobacterial mce4 locus encodes a steroid transporter. J Biol Chem 2008, 283:35368-35374.

32. Casali N, Riley LW: A phylogenomic analysis of the actinomycetales mce operons. BMC Genomics 2007, 8:60

33. Kumar A, Chandolia A, Chaudhry U, Brahmachari V, Bose M: Comparison of mammalian cell entry operons of mycobacteria: in silico analysis and expression profiling. FEMS Immunol Med Microbiol 2005, 43:185-195.

34. Beaman BL, Beaman L: Nocardia species: host-parasite relationships. Clin Microbiol Rev 1994, 7:213-264.

35. Matsunaga I, Naka T, Talekar RS, McConnell MJ, Katoh K, Nakao H, Otsuka A, Behar SM, Yano I, Moody DB, Sugita M: Mycolyltransferase-mediated glycolipid exchange in mycobacteria. J Biol Chem 2008, 283:28835-28841.

36. Spargo BJ, Crowe LM, loneda T, Beaman BL, Crowe JH: Cord factor (alpha, alpha-trehalose 6,6'-dimycolate) inhibits fusion between phospholipid vesicles. Proc Natl Acad Sci U S A 1991, 88:737-740.

37. Tonge PJ: Another brick in the wall. Nat Struct Biol 2000, 7:94-96.

38. Camp DM, Loeffler DA, Razoky BA, Tam S, Beaman BL, LeWitt PA: Nocardia asteroides culture filtrates cause dopamine depletion and cytotoxicity in PC12 cells. Neurochem Res 2003, 28:1359-1367.

39. Emeruwa AC: Isolation and some properties of beta-hemolysin produced by Nocardia asteroides. Mycopathologia 1986, 95:29-35.

40. Li JM, Li N, Zhu DY, Wan LG, He YL, Yang C: Isocitrate lyase from Mycobacterium tuberculosis promotes survival of Mycobacterium smegmatis within macrophage by suppressing cell apoptosis. Chin Med J (Engl) 2008, 121:1114-1119.

41. Ishikawa J, Yamashita A, Mikami Y, Hoshino Y, Kurita H, Hotta K, Shiba T, Hattori M: The complete genomic sequence of Nocardia farcinica IFM 10152. Proc Natl Acad Sci U S A 2004, 101:14925-14930.

42. Stinear TP, Seemann T, Harrison PF, Jenkin GA, Davies JK, Johnson PD, Abdellah Z, Arrowsmith C, Chillingworth T, Churcher C, et al: Insights from the complete genome sequence of Mycobacterium marinum on the evolution of Mycobacterium tuberculosis. Genome Res 2008, 18:729-741.

43. Sultana R, Tanneeru K, Guruprasad L: The PE-PPE domain in Mycobacterium reveals a serine alpha/beta hydrolase fold and function: an in-silico analysis. PLoS One 2011, 6:e16745.

44. Beaman BL, Scates SM, Moring SE, Deem R, Misra HP: Purification and properties of a unique superoxide dismutase from Nocardia asteroides. J Biol Chem 1983, 258:91-96.

45. Nesbit EA, Gunasekaran M: Influence of cultural conditions on growth and lipolytic activity in Nocardia asteroides. Folia Microbiol (Praha) 1993, 38:451-455.

46. Zlotnik H, Schramm VL, Buckley HR: Purification and partial characterization of a Nocardia brasiliensis extracellular protease. J Bacteriol 1984, 157:627-631.

47. Brodin P, Rosenkrands I, Andersen P, Cole ST, Brosch R: ESAT- 6 proteins: protective antigens and virulence factors? Trends Microbiol 2004 12:500-508.

48. Murakami Y, Kato S, Nakajima M, Matsuoka M, Kawai H, Shin-Ya K, Seto H: Formobactin, a novel free radical scavenging and neuronal cell protecting substance from Nocardia sp. J Antibiot (Tokyo) 1996, 49:839-845.
49. Suenaga K, Kokubo S, Shinohara C, Tsuji T, Uemura D: Structures of amistatins $A$ and $B$, novel growth inhibitors of human tumor cell lines from an actinomycete. Tetrahedron letters 1999, 40:1945-1948.

50. Tsuda M, Yamakawa M, Oka S, Tanaka Y, Hoshino Y, Mikami Y, Sato A, Fujiwara H, Ohizumi $Y$, Kobayashi J: Brasilibactin A, a cytotoxic compound from actinomycete Nocardia brasiliensis. J Nat Prod 2005, 68:462-464

51. Nemoto A, Hoshino Y, Yazawa K, Ando A, Mikami Y, Komaki H, Tanaka Y, Grafe U: Asterobactin, a new siderophore group antibiotic from Nocardia asteroides. J Antibiot (Tokyo) 2002, 55:593-597.

52. Hoshino $Y$, Chiba K, Ishino K, Fukai T, Igarashi $Y$, Yazawa K, Mikami $Y$, Ishikawa J: Identification of nocobactin NA biosynthetic gene clusters in Nocardia farcinica. J Bacteriol 2011, 193:441-448.

53. Hanada M, Sugawara K, Kaneta K, Toda S, Nishiyama Y, Tomita K, Yamamoto $\mathrm{H}$, Konishi M, Oki T: Epoxomicin, a new antitumor agent of microbial origin. J Antibiot (Tokyo) 1992, 45:1746-1752.

54. Mukai A, Fukai T, Matsumoto Y, Ishikawa J, Hoshino Y, Yazawa K, Harada K, Mikami Y: Transvalencin Z, a new antimicrobial compound with salicylic acid residue from Nocardia transvalensis IFM 10065. J Antibiot (Tokyo) 2006, 59:366-369.

55. Espinoza-Gonzalez NA, Welsh O, de Torres NW, Cavazos-Rocha N, OcampoCandiani J, Said-Fernandez S, Lozano-Garza G, Choi SH, Vera-Cabrera L: Efficacy of DA-7218, a new oxazolidinone prodrug, in the treatment of experimental actinomycetoma produced by Nocardia brasiliensis. Molecules 2008, 13:31-40.

56. Mukai A, Fukai T, Hoshino Y, Yazawa K, Harada K, Mikami Y: Nocardithiocin, a novel thiopeptide antibiotic, produced by pathogenic Nocardia pseudobrasiliensis IFM 0757. J Antibiot (Tokyo) 2009, 62:613-619.

57. Alegre MT, Cournoyer B, Mesas JM, Guerineau M, Normand P, Pernodet JL: Cloning of Frankia species putative tRNA(Pro) genes and their efficacy for pSAM2 site-specific integration in Streptomyces lividans. Appl Environ Microbiol 1994, 60:4279-4283.

58. Yao W, Yang Y, Chiao J: IS204: an insertion sequence from Nocardia asteroides (mexicana) YP21. Plasmid 1994, 32:262-269.

59. Bickhart DM, Gogarten JP, Lapierre P, Tisa LS, Normand P, Benson DR: Insertion sequence content reflects genome plasticity in strains of the root nodule actinobacterium Frankia. BMC Genomics 2009, 10:468.

60. Graindorge A, Menard A, Monnez C, Cournoyer B: Insertion sequence evolutionary patterns highlight convergent genetic inactivations and recent genomic island acquisitions among epidemic Burkholderia cenocepacia. J Med Microbiol 2012, 61:394-409.

61. Konstantinidis KT, Tiedje JM: Trends between gene content and genome size in prokaryotic species with larger genomes. Proc Natl Acad Sci U S A 2004, 101:3160-3165.

62. Song H, Hwang J, Yi H, Ulrich RL, Yu Y, Nierman WC, Kim HS: The early stage of bacterial genome-reductive evolution in the host. PLoS Pathog 2010, 6:e1000922.

63. Zoropogui A, Pujic P, Normand P, Barbe V, Beaman B, Beaman L, Boiron P, Colinon C, Deredjian A, Graindorge A, et al: Genome sequence of the human- and animal-pathogenic strain Nocardia cyriacigeorgica GUH-2. J Bacteriol 2012, 194:2098-2099.

64. Vallenet D, Engelen S, Mornico D, Cruveiller S, Fleury L, Lajus A, Rouy Z, Roche D, Salvignol G, Scarpelli C, Medigue C: MicroScope: a platform for microbial genome annotation and comparative genomics. Database (Oxford) 2009, 2009:bap021.

65. Boyer F, Morgat A, Labarre L, Pothier J, Viari A: Syntons, metabolons and interactons: an exact graph-theoretical approach for exploring neighbourhood between genomic and functional data. Bioinformatics 2005, 21:4209-4215.

66. Vernikos GS, Parkhill J: Interpolated variable order motifs for identification of horizontally acquired DNA: revisiting the Salmonella pathogenicity islands. Bioinformatics 2006, 22:2196-2203.

67. Waack S, Keller O, Asper R, Brodag T, Damm C, Fricke WF, Surovcik K, Meinicke $P$, Merkl R: Score-based prediction of genomic islands in prokaryotic genomes using hidden Markov models. BMC Bioinforma 2006, 7:142.

68. Carver TJ, Rutherford KM, Berriman M, Rajandream MA, Barrell BG, Parkhill J: ACT: the Artemis comparison tool. Bioinformatics 2005, 21:3422-3423.

69. R_Development_Core_Team: $R$ : a language and environment for statistical computing. Vienna, Austria; 2011.

70. Sorensen AL, Nagai S, Houen G, Andersen P, Andersen AB: Purification and characterization of a low-molecular-mass T-cell antigen secreted by Mycobacterium tuberculosis. Infect Immun 1995, 63:1710-1717. 
71. Gordon SV, Brosch R, Billault A, Garnier T, Eiglmeier K, Cole ST: Identification of variable regions in the genomes of tubercle bacilli using bacterial artificial chromosome arrays. Mol Microbiol 1999, 32:643-655.

72. Mastronunzio JE, Tisa LS, Normand P, Benson DR: Comparative secretome analysis suggests low plant cell wall degrading capacity in Frankia symbionts. BMC Genomics 2008, 9:47.

73. Gardy JL, Brinkman FS: Methods for predicting bacterial protein subcellular localization. Nat Rev Microbiol 2006, 4:741-751.

74. Sonnhammer EL, Eddy SR, Birney E, Bateman A, Durbin R: Pfam: multiple sequence alignments and HMM-profiles of protein domains. Nucleic Acids Res 1998, 26:320-322.

75. Nogueira T, Rankin DJ, Touchon M, Taddei F, Brown SP, Rocha EP. Horizontal gene transfer of the secretome drives the evolution of bacterial cooperation and virulence. Curr Biol 2009, 19:1683-1691.

doi:10.1186/1471-2164-14-286

Cite this article as: Zoropogui et al:: The Nocardia cyriacigeorgica GUH-2 genome shows ongoing adaptation of an environmental Actinobacteria to a pathogen's lifestyle. BMC Genomics 2013 14:286.

\section{Submit your next manuscript to BioMed Central and take full advantage of:}

- Convenient online submission

- Thorough peer review

- No space constraints or color figure charges

- Immediate publication on acceptance

- Inclusion in PubMed, CAS, Scopus and Google Scholar

- Research which is freely available for redistribution 